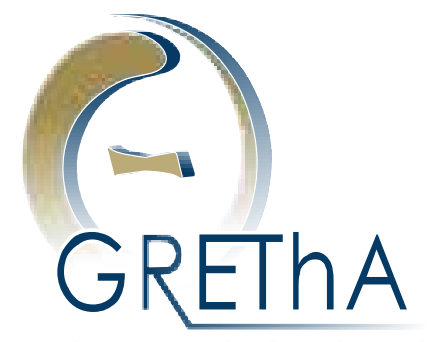

Groupe de Recherche en

Économie Théorique et Appliquée

\title{
Rearrangements and Sequential Rank Order Dominance
}

\author{
Patrick MOYES \\ GREThA, CNRS, UMR 5113 \\ Université de Bordeaux \\ \& \\ IDEP MARSEILLE
}

Cahiers du GREThA

$n^{\circ}$ 2013-10

March

\author{
GRETHA UMR CNRS 5113 \\ Université Montesquieu Bordeaux IV \\ Avenue Léon Duguit - 33608 PESSAC - FRANCE \\ Tel : +33 (0)5.56.84.25.75 - Fax : +33 (0)5.56.84.86.47 - www.gretha.fr
}




\title{
Réarrangements et Dominance en Quantiles Séquentielle*
}

Résumé

L'analyse distributive implique généralement des comparaisons de distributions où les individus se distinguent sur la base de plusieurs attributs. Dans le cas particulier où il y a deux attributs et où la distribution de l'un de ces deux attributs est fixée, on peut faire appel au critère de la dominance en quantiles séquentielle afin de comparer les distributions. Nous montrons que, si une distribution est classée au-dessus d'une autre par le critère de la dominance en quantiles séquentielle, alors la distribution dominante peut être obtenue à partir de la distribution dominée au moyen d'une suite finie de permutations favorables, et réciproquent. Nous présentons deux exemples où les permutations favorables se révèlent avoir des implications intéressantes d'un point de vue normatif.

Mots-clés : Réarrangements, Permutations Favorables, Dominance en Quantiles Séquentielle, Inégalité, Appariement, Mobilité

\section{Rearrangements and Sequential Rank Order Dominance*}

\begin{abstract}
Distributive analysis typically involves comparisons of distributions where individuals differ in more than just one attribute. In the particular case where there are two attributes and where the distribution of one of these two attributes is fixed, one can appeal to sequential rank order dominance for comparing distributions. We show that sequential rank order domination of one distribution over another implies that the dominating distribution can be obtained from the dominated one by means of a finite sequence of favourable permutations, and conversely. We provide two examples where favourable permutations prove to have interesting implications from a normative point of view.
\end{abstract}

Keywords: Rearrangements, Favourable Permutations, Sequential Rank Order Dominance, Inequality, Matching, Mobility.

JEL: D30, D63, 132

Reference to this paper: MOYES Patrick (2013) Rearrangements and Sequential Rank Order Dominance, Cahiers du GREThA, n²013-10.

http://ideas.repec.org/p/grt/wpegrt/2013-10.html.

* This paper forms part of the research project The Multiple Dimensions of Inequality (Contract No.ANR 2010 BLANC 1808) of the French National Agency for Research, whose financial support is ratefully acknowledged. I am indebted to Stephen Bazen, Nicolas Gravel, François Maniquet, the Editor and two anonymous referees for very useful comments and suggestions when preparing this version. Needless to say, none of the persons mentioned above should be held responsible for remaining deficiencies. 


\section{Introduction}

The notion of a progressive transfer has long been known to play a crucial role when one is interested in the distribution of a single cardinal attribute like income. Transferring part of the income of a rich individual to a poorer one in such a way that their relative positions are unchanged reduces inequality in the sense that the Lorenz curve moves upwards. In other words, the total income possessed by any fraction of the population consisting of the poorest individuals increases as the result of a progressive transfer. More interestingly, it can been shown that the converse statement also holds: if one distribution is ranked above another by the Lorenz criterion, then it can be derived from the latter by a finite sequence of progressive transfers. This result has important implications from a normative point of view because the Lorenz ranking of distributions is identical to the ranking implied by utilitarian unanimity over the class of concave utility functions. Following its proof by Hardy, Littlewood, and Pólya (1934), the equivalence between the three above statements is known as the Hardy-LittlewoodPólya theorem (see also Berge (1963), Dasgupta, Sen, and Starrett (1973), Marshall and Olkin (1979) among others).

However the ability of income alone to measure a person's well-being has been challenged during the last thirty years and there has been an increasing concern for a more comprehensive approach involving different dimensions of individual well-being. Considering distributions of two cardinal attributes, Atkinson and Bourguignon (1982) have demonstrated that utilitarian unanimity over the class of utility functions with non-positive cross-derivatives - the socalled submodular functions - ranks distributions in the same way as first degree bidimensional stochastic dominance. When one of the two attributes is of an ordinal nature and when in addition its marginal distribution is fixed, Atkinson and Bourguignon (1987) have shown that bidimensional first degree stochastic dominance reduces to sequential rank order dominance. To illustrate things, consider the case where every individual in the population is identified by her health achievement and her income. Suppose further that an individual's health achievement falls into a finite set of ordered categories like "poor", "fair", "good", "very good", and "excellent" as it is typically the case in self-reporting questionnaires. The sequential rank order criterion consists in comparing, first the quantile distributions of income for those individuals with "poor" health, then the quantile distributions of income for those individuals in the combined "poor" or "fair" health categories, and so on until all health categories have been taken into account. If one distribution is ranked above another at each stage of this process, then it is declared to be better according to the sequential rank order criterion.

First degree bidimensional stochastic dominance is based on the comparisons of the proportion of individuals who are deprived in the two attributes of interest. According to this criterion, one distribution dominates another if, for all possible two-dimensional thresholds, there are fewer deprived individuals in the former distribution than in the latter. Like the Lorenz criterion, the sequential rank order criterion builds on the distributions of income, but it recognises the importance of the second variable - health achievement in the discussion above - through the way the distributions of the different categories are assembled. By making 
explicit the interplay between the distribution of income within categories and the distribution of individuals across categories, the sequential rank order criterion may be considered a more transparent criterion than the standard bidimensional stochastic dominance, admittedly in the case where the marginal distributions of the two variables are fixed. Or at least, it provides a different perspective that complements that given by standard first degree bidimensional stochastic dominance.

However, the two above mentioned articles failed to identify those elementary transformations that play a role similar to progressive transfers in a bidimensional framework. Admittedly, Atkinson and Bourguignon (1982) have suggested that correlation decreasing transformations might be the analogue of progressive transfers when distributions are ordered by first degree stochastic dominance or equivalently by the rank order criterion. Indeed, it is rather natural in a bidimensional context to consider that the more correlated the two variables, the more unequal the situation is. Then, any transfer of densities that leaves unchanged the marginal distributions and decreases the correlation between the two attributes may be considered an improvement. Epstein and Tanny (1980) and Tchen (1980) must be credited for having shown that first degree bidimensional stochastic domination of one distribution by another is equivalent to the fact that the dominating distribution can be obtained from the dominated one through successive applications of such correlation decreasing transformations. By combining these different results, one obtains an equivalence between three statements - namely, utilitarian unanimity over the class of submodular utility functions, bidimensional first degree stochastic dominance, and appropriate combinations of correlation decreasing transformations - that is very close to the Hardy-Littlewood-Pólya theorem described above.

The starting point of the present paper is the recognition that the notion of a correlation decreasing transformation is defined by reference to the joint distribution function. As a consequence, to implement the algorithm designed by Epstein and Tanny (1980) and Tchen (1980) one must know what the joint distribution functions look like. It might be interesting to know what is the counterpart of a correlation decreasing transformation if we wanted to construct an algorithm allowing us to derive the dominating distribution from the dominated distribution starting now with the sequential quantile curves rather than the joint distribution functions. Since the sequential rank order dominance and first degree stochastic dominance coincide when the marginal distributions are fixed, one might object that it makes little sense to search for such an algorithm: if one distribution dominates another according to the sequential rank order criterion, then it can be obtained from the latter by means of correlation decreasing transformations. However, the meaning of a correlation decreasing transformation is far from being clear when we are interested in the comparison of bidimensional distributions for populations consisting of a finite number of individuals. For in this case we face the constraint that the densities that are moved on the two-dimensional grid defined by the distinct values of the two attributes belong to the set of rational numbers. In the limit - and in order to make things more transparent - we might even want that the densities that are transferred be all equal to one over $n$ assuming that there are $n$ individuals in the population. 
Moyes (2012) has suggested that favourable permutations might be the elementary transformations that play a similar role as progressive transfers for sequential rank order dominance. Given two individuals such that one is (strictly) more deprived than the other in both attributes - for instance, income and health - a favourable permutation consists in exchanging the incomes of the two individuals so that the individual in bad health is given the income of the individual in better health who is also the richer. Then, it can be easily shown that favourable permutations always result in an increase in social welfare when it is evaluated by the utilitarian rule and when the utility function is submodular (see, e.g., Moyes (2012)). Admittedly, a favourable permutation is a particular case of a correlation decreasing transformation where the amount of density transferred is equal to one over some integer and it may be considered to some extent a more primitive concept.

The organisation of the note is as follows. We devote Section 2 to the simple case where a single attribute is distributed among individuals and we introduce preliminary results that will prove to be useful later on. We examine the case of the distribution of two attributes - one of which is cardinal and the other one ordinal - in Section 3. We present and prove our main result according to which sequential rank order domination of one distribution by another implies that the dominating distribution can be obtained from the dominated one by means of successive favourable permutations. We review in Section 4 two examples that involve favourable permutations and where the application of the sequential rank order criterion proves to be of some interest. Section 5 concludes the paper pointing at limitations and possible extensions. Finally, we provide in Section A an illustration of the algorithm used in the proof of our main result by means of a simple example.

\section{Unidimensional Distributions and Preliminary Results}

To begin with, it is convenient to consider the case of distributions of a single attribute for a fixed and homogeneous population of individuals $N:=\{1,2, \ldots, n\}$ with $n \geqq 2$. A distribution for population $N$ is a list $\mathbf{u}:=\left(u_{1}, \ldots, u_{n}\right)$, where $u_{i} \in \mathscr{D} \subset \mathbb{R}$ may be viewed as the income of individual $i$, but other interpretations are also possible.

Definition 2.1. Given two income distributions $\mathbf{u}:=\left(u_{1}, \ldots, u_{n}\right), \mathbf{v}:=\left(v_{1}, \ldots, v_{n}\right) \in \mathscr{D}^{n}$, we say that $\mathbf{u}$ component-wise dominates $\mathbf{v}$, which we write $\mathbf{u} \geq \mathbf{v}$, if and only if:

$$
u_{h} \geqq v_{h}, \forall h=1,2, \ldots, n \text {. }
$$

We denote respectively by $\sim$ and $>$ the symmetric and asymmetric components of $\geq$ defined in the usual way, and we further note that $\mathbf{u} \sim \mathbf{v}$ if and only if $u_{h}=v_{h}$, for all $h=1,2, \ldots, n$. The non-decreasing rearrangement of an income distribution $\mathbf{u}:=\left(u_{1}, \ldots, u_{n}\right) \in \mathscr{D}^{n}$ is indicated by $\tilde{\mathbf{u}}:=\left(\tilde{u}_{1}, \tilde{u}_{2}, \ldots, \tilde{u}_{n}\right)$, where $\tilde{u}_{1} \leqq \tilde{u}_{2} \leqq \cdots \leqq \tilde{u}_{n}$.

Definition 2.2. Given two income distributions $\mathbf{u}:=\left(u_{1}, \ldots, u_{n}\right), \mathbf{v}:=\left(v_{1}, \ldots, v_{n}\right) \in \mathscr{D}^{n}$, we say that $\mathbf{u}$ rank order dominates $\mathbf{v}$, which we write $\mathbf{u} \geq_{R O} \mathbf{v}$, if and only if:

$$
\tilde{u}_{h} \geqq \tilde{v}_{h}, \forall h=1,2, \ldots, n .
$$


The symmetric and asymmetric components of $\geq_{R O}$ are indicated by $\sim_{R O}$ and $>_{R O}$, respectively, and we note that $\mathbf{u} \sim_{R O} \mathbf{v}$ if and only if $\tilde{u}_{h}=\tilde{v}_{h}$, for all $h=1,2, \ldots, n$, in which case $\mathbf{u}$ is a permutation of $\mathbf{v}$. Component-wise dominance implies rank-order dominance, but the converse implication is false as the next result demonstrates.

Lemma 2.1. Let $\mathbf{u}, \mathbf{v} \in \mathscr{D}^{n}$ and consider the following two statements:

(a) $\mathbf{u} \geq[>] \mathbf{v}$.

(b) $\mathbf{u} \geq_{R O}\left[>_{R O}\right] \mathbf{v}$.

Then, statement (a) implies statement (b), but the converse implication does not hold.

\section{Proof.}

(a) $\Longrightarrow$ (b). By definition, we have $u_{h} \geqq v_{h}$, for all $h \in\{1,2, \ldots, n\}$. Consider the indices $i_{1}$ and $j_{1}$ defined by $u_{i_{1}} \geqq u_{h}$, for all $h \in\{1,2, \ldots, n\}$, and $v_{j_{1}} \geqq v_{h}$, for all $h \in\{1,2, \ldots, n\}$.

CASE 1: $i_{1}=j_{1}$. Then $\tilde{u}_{n}=u_{i_{1}} \geqq v_{i_{1}}=\tilde{v}_{n}$ and $u_{h} \geqq v_{h}$, for all $h \in\{1,2, \ldots, n\} \backslash\left\{i_{1}\right\}$. Let

$$
\begin{aligned}
\mathbf{u}^{1} & :=\left(u_{1}, \ldots, u_{i_{1}-1}, u_{i_{1}+1}, \ldots, u_{n}\right) \\
\mathbf{v}^{1} & :=\left(v_{1}, \ldots, v_{i_{1}-1}, v_{i_{1}+1}, \ldots, v_{n}\right) \\
\hat{\mathbf{u}}^{1} & :=\left(\tilde{u}_{n}\right)=\left(u_{i_{1}}\right) \\
\hat{\mathbf{v}}^{1} & :=\left(\tilde{v}_{n}\right)=\left(v_{i_{1}}\right)
\end{aligned}
$$

Then, we have $\mathbf{u}^{1} \geq \mathbf{v}^{1}$ and $\hat{\mathbf{u}}^{1} \geq \hat{\mathbf{v}}^{1}$.

CASE 2: $i_{1}<j_{1}$. We have $u_{i_{1}} \geqq u_{j_{1}} \geqq v_{j_{1}} \geqq v_{i_{1}}$, from which we deduce that $\tilde{u}_{n}=u_{i_{1}} \geqq v_{j_{1}}=$ $\tilde{v}_{n}$ and $u_{j_{1}} \geqq v_{i_{1}}$. In addition, $u_{h} \geqq v_{h}$, for all $h \in\{1,2, \ldots, n\} \backslash\left\{i_{1}, j_{1}\right\}$. Denote as $\mathbf{v}^{*}$ the permutation of distribution $\mathbf{v}$ defined by

$$
\mathbf{v}^{*}:=\left(v_{1}, \ldots, v_{i_{1}-1}, v_{j_{1}}, v_{i_{1}+1}, \ldots, v_{j_{1}-1}, v_{i_{1}}, v_{j_{1}+1}, \ldots, v_{n}\right) \text {. }
$$

From what precedes, we deduce that $\mathbf{u} \geq \mathbf{v}^{*}$. Define next

$$
\begin{aligned}
\mathbf{u}^{1} & :=\left(u_{1}, \ldots, u_{i_{1}-1}, u_{i_{1}+1}, \ldots, u_{j_{1}}, \ldots, u_{n}\right) \\
\mathbf{v}^{1} & :=\left(v_{1}^{*}, \ldots, v_{i_{1}-1}^{*}, v_{i_{1}+1}^{*}, \ldots, v_{j_{1}}^{*} \ldots, v_{n}^{*}\right)=\left(v_{1}, \ldots, v_{i_{1}-1}, v_{i_{1}+1}, \ldots, v_{i_{1}}, \ldots, v_{n}\right) \\
\hat{\mathbf{u}}^{1} & :=\left(\tilde{u}_{n}\right)=\left(u_{i_{1}}\right) \\
\hat{\mathbf{v}}^{1} & :=\left(\tilde{v}_{n}\right)=\left(v_{j_{1}}\right) .
\end{aligned}
$$

Then, we obtain $\mathbf{u}^{1} \geq \mathbf{v}^{1}$ and $\hat{\mathbf{u}}^{1} \geq \hat{\mathbf{v}}^{1}$.

CASE 3: $i_{1}>j_{1}$. One can prove using a similar argument to that above that $\mathbf{u}^{1} \geq \mathbf{v}^{1}$ and $\hat{\mathbf{u}}^{1} \geq \hat{\mathbf{v}}^{1}$.

By applying the above reasoning to the distributions $\mathbf{u}^{1}$ and $\mathbf{v}^{1}$, we construct distributions $\mathbf{u}^{2}, \mathbf{v}^{2}, \hat{\mathbf{u}}^{2}:=\left(\tilde{u}_{n-1}, \tilde{u}_{n}\right)$, and $\hat{\mathbf{v}}^{2}:=\left(\tilde{v}_{n-1}, \tilde{v}_{n}\right)$ such that $\mathbf{u}^{2} \geq \mathbf{v}^{2}$ and $\hat{\mathbf{u}}^{2} \geq \hat{\mathbf{v}}^{2}$. By successive 
application of this reasoning, we finally obtain two distributions $\hat{\mathbf{u}}^{n}=\tilde{\mathbf{u}}$ and $\hat{\mathbf{v}}^{n}=\tilde{\mathbf{v}}$ such that $\hat{\mathbf{u}}^{n} \geq \hat{\mathbf{v}}^{n}$, i.e., $\mathbf{u} \geq_{R O} \mathbf{v}$. A slight adaptation of the proof above allows one to establish that $\mathbf{u}>\mathbf{v}$ implies $\mathbf{u}>_{R O} \mathbf{v}$.

$\neg[(\mathrm{a}) \Longrightarrow(\mathrm{b})]$ : Choose $\mathbf{u}:=(4,2,2)$ and $\mathbf{v}:=(1,1,3)$, which are not comparable according to the component-wise ordering, though $\tilde{\mathbf{u}}:=(2,2,4)>(1,1,3)=: \tilde{\mathbf{v}}$.

The next lemma is well-known (see, e.g., Saposnik (1981)) and it simply states that the ranking of income distributions by utilitarian unanimity over the class of non-decreasing utility functions is equivalent to the ranking implied by the rank order criterion. More precisely, letting

$$
\Phi:=\{\phi: \mathscr{D} \rightarrow \mathbb{R} \mid \phi \text { is non-decreasing }\}
$$

we have the following result:

Lemma 2.2. Let $\mathbf{u}, \mathbf{v} \in \mathscr{D}^{n}$. Then, statements (a) and (b) below are equivalent:

(a) $\sum_{h=1}^{n} \phi\left(u_{h}\right) \geqq \sum_{h=1}^{n} \phi\left(v_{h}\right), \forall \phi \in \Phi$.

(b) $\mathbf{u} \geq_{R O} \mathbf{v}$.

This result mirrors the standard equivalence between first degree stochastic dominance and utilitarian unanimity (see, e.g., Fishburn and Vickson (1978)).

\section{Heterogenous Distributions and Sequential Rank Order Dominance}

From now on, we assume that individuals can be distinguished on the basis of two attributes where the first one can be assimilated with income as above and where the second is a categorical variable which allows us to partition the population into a finite number of groups of similar - with respect to that attribute - individuals. A good example of the latter attribute is health achievement which typically takes a finite number of modalities and defines as many categories or types of individuals. More generally, any variable that determines a natural linear order and that can be associated with income constitutes a potential candidate for our second attribute. We denote by $\mathscr{H}:=\{1,2, \ldots, H\}$ the set of categories or types with the convention that - other things equal - an individual of type $h+1$ is in a better situation than an individual of type $h$.

It is then natural to conceive of a bidimensional distribution or situation as a couple $(\mathbf{x} ; \mathbf{a}):=$ $\left(x_{1}, \ldots, x_{n} ; a_{1}, \ldots, a_{n}\right) \in \mathscr{D}^{n} \times \mathscr{H}^{n}$ such that $\left(x_{i}, a_{i}\right)$ fully describes individual $i$, where $x_{i} \in \mathscr{D}$ and $a_{i} \in \mathscr{H}$ are respectively the income and the type of individual $i$. Then, we associate with $(\mathbf{x} ; \mathbf{a}) \in \mathscr{D}^{n} \times \mathscr{H}^{n}$ the heterogeneous distribution $\mathbf{u}:=\left(\mathbf{u}^{1} ; \ldots ; \mathbf{u}^{H}\right)$, where

$$
\mathbf{u}^{h}:=\left(u_{1}^{h}, u_{2}^{h}, \ldots, u_{n(h)}^{h}\right)
$$

is the income distribution of the subpopulation consisting of all the individuals of type $h$, $n(h):=\# N(h) \geqq 1$, and $N(h):=\left\{i \in\{1,2, \ldots, n\} \mid a_{i}=h\right\}$, for all $h \in \mathscr{H}$. Without loss 
of generality, we assume that incomes within populations of individuals of the same type are non-decreasingly arranged so that

$$
u_{1}^{h} \leqq u_{2}^{h} \leqq \cdots \leqq u_{n(h)}^{h}
$$

We indicate by $\mathbf{u}^{(h)}:=\left(\mathbf{u}^{1} ; \mathbf{u}^{2} ; \ldots ; \mathbf{u}^{h}\right)$ the distribution of the incomes received by the individuals with types equal to $h$ or less and we note that $\mathbf{u}^{(H)}=\mathbf{u}$. We let

$$
\tilde{\mathbf{u}}^{(h)}:=\left(\tilde{u}_{1}^{(h)}, \tilde{u}_{2}^{(h)}, \ldots, \tilde{u}_{\tilde{n}(h)-1}^{(h)}, \tilde{u}_{\tilde{n}(h)}^{(h)}\right)
$$

stand for the non-decreasing rearrangement of $\mathbf{u}^{(h)}$ defined by

$$
\tilde{u}_{1}^{(h)} \leqq \tilde{u}_{2}^{(h)} \leqq \cdots \leqq \tilde{u}_{\tilde{n}(h)-1}^{(h)} \leqq \tilde{u}_{\tilde{n}(h)}^{(h)},
$$

where $\tilde{n}(h):=\sum_{k=1}^{h} n(k)$. Consider two situations $\left(\mathbf{x}^{*} ; \mathbf{a}^{*}\right)$ and $\left(\mathbf{x}^{\circ} ; \mathbf{a}^{\circ}\right)$ such that $\mathbf{a}^{*}=\mathbf{a}^{\circ}$, which implies that $n^{*}(h)=n^{\circ}(h)=n(h)$, for all $h \in \mathscr{H}$ : the number of individuals of type $h$ in the two situations is the same. Situations $\left(\mathbf{x}^{*} ; \mathbf{a}^{*}\right)$ and $\left(\mathbf{x}^{\circ} ; \mathbf{a}^{\circ}\right)$ only differ with respect to the way income is distributed among the types. Denote by $\mathbf{u}$ and $\mathbf{v}$ the heterogeneous distributions associated with the situations $\left(\mathbf{x}^{*} ; \mathbf{a}^{*}\right)$ and $\left(\mathbf{x}^{\circ} ; \mathbf{a}^{\circ}\right)$, respectively.

It will greatly simplify the proofs if individuals, whatever their types, have different incomes in the situations under comparison. Given $\mathbf{u}:=\left(\mathbf{u}^{1} ; \ldots ; \mathbf{u}^{H}\right), \mathbf{v}:=\left(\mathbf{v}^{1} ; \ldots ; \mathbf{v}^{H}\right) \in \mathscr{D}^{n(1)} \times \cdots \times$ $\mathscr{D}^{n(H)}$, we define:

$$
\begin{aligned}
& \mathscr{H}_{0}:=\left\{h \in \mathscr{H} \mid u_{g}^{h}=v_{g}^{h}, \forall g \in N(h)\right\} ; \\
& \mathscr{H}_{1}:=\left\{h \in \mathscr{H} \mid u_{g}^{h} \neq v_{g}^{h}, \exists g \in N(h)\right\} ; \\
& S(h):=\left\{g \in N(h) \mid u_{g}^{h}=v_{g}^{h}\right\} \text { for } h \in \mathscr{H}_{1} ; \\
& T(h):=\left\{g \in N(h) \mid u_{g}^{h} \neq v_{g}^{h}\right\} \text { for } h \in \mathscr{H}_{1} ;
\end{aligned}
$$

and we note that $\varnothing \subseteq S(h) \subset N(h), \varnothing \subset T(h) \subseteq N(h)$, and $S(h) \cup T(h)=N(h)$, for all $h \in \mathscr{H}_{1}$. Consider the following class of $H$-tuples of utility functions:

$$
\boldsymbol{\Psi}^{*}:=\left\{\boldsymbol{\psi}:=\left(\psi_{1}, \ldots, \psi_{H}\right) \mid \psi_{h}(y) \text { is continuous in } y, \forall h=1,2, \ldots, H\right\}
$$

where $\psi_{h}(y)$ is the utility derived from income $y$ by an individual of type $h .{ }^{1}$ Given the $H$ tuple $\boldsymbol{\psi}:=\left(\psi_{1}, \ldots, \psi_{H}\right) \in \boldsymbol{\Psi}^{\circ} \subseteq \boldsymbol{\Psi}^{*}$, we denote by $\boldsymbol{\psi}\left(\mathscr{H}_{1}\right):=\left(\left(\psi_{h}\right)_{h \in \mathscr{H}_{1}}\right)$ its restriction to $\mathscr{H}_{1}$ and by $\Psi^{\circ}\left(\mathscr{H}_{1}\right)$ the set of such profiles. Then, we have the following obvious result:

Lemma 3.1. Let $\mathbf{u}:=\left(\mathbf{u}^{1} ; \ldots ; \mathbf{u}^{H}\right), \mathbf{v}:=\left(\mathbf{v}^{1} ; \ldots ; \mathbf{v}^{H}\right) \in \mathscr{D}^{n(1)} \times \cdots \times \mathscr{D}^{n(H)}$ and $\Psi^{\circ} \subseteq \Psi^{*}$. Then, statements (a) and (b) below are equivalent:

(a) $\sum_{h=1}^{H} \sum_{i=1}^{n(h)} \psi_{h}\left(u_{i}^{h}\right) \geqq \sum_{h=1}^{H} \sum_{i=1}^{n(h)} \psi_{h}\left(v_{i}^{h}\right)$, for all $\boldsymbol{\psi}:=\left(\psi_{1}, \ldots, \psi_{H}\right) \in \boldsymbol{\Psi}^{\circ}$.

\footnotetext{
${ }^{1}$ Imposing that the utility functions are continuous is not constraining to the extent that it is always possible to approximate a discontinuous function by a continuous one as long as the number of points of discontinuity is finite (see, e.g., Fishburn and Vickson (1978).
} 
(b) $\sum_{h \in \mathscr{H}_{1}} \sum_{i \in T(h)} \psi_{h}\left(u_{i}^{h}\right) \geqq \sum_{h \in \mathscr{H}_{1}} \sum_{i \in T(h)} \psi_{h}\left(v_{i}^{h}\right)$, for all $\boldsymbol{\psi}\left(\mathscr{H}_{1}\right) \in \boldsymbol{\Psi}^{\circ}\left(\mathscr{H}_{1}\right)$.

According to Lemma 3.1, ruling out unconcerned individuals - those whose situations are identical in the distributions under comparison - does not change the ranking of these distributions by utilitarian unanimity. Quite interestingly, this result does not require that particular restrictions be placed on the $H$-tuples $\boldsymbol{\psi}:=\left(\psi_{1}, \ldots, \psi_{H}\right)$ of utility functions.

In order to compare heterogeneous distributions we make recourse to the sequential rank order dominance criterion introduced by Atkinson and Bourguignon (1987) for which we give a formal definition below.

Definition 3.1. Given two heterogeneous distributions $\mathbf{u}:=\left(\mathbf{u}^{1} ; \ldots ; \mathbf{u}^{H}\right), \mathbf{v}:=\left(\mathbf{v}^{1} ; \ldots ; \mathbf{v}^{H}\right) \in$ $\mathscr{D}^{n(1)} \times \cdots \times \mathscr{D}^{n(H)}$, we say that $\mathbf{u}$ sequential rank order dominates $\mathbf{v}$, which we write $\mathbf{u} \geq_{S R O} \mathbf{v}$, if and only if:

$$
\mathbf{u}^{(h)} \geq_{R O} \mathbf{v}^{(h)}, \forall h=1,2, \ldots, H-1, \text { and } \mathbf{u}^{(H)} \sim_{R O} \mathbf{v}^{(H)} .
$$

If in addition $\mathbf{u}^{(k)}>_{R O} \mathbf{v}^{(k)}$, for some $k<H$, then we say that $\mathbf{u}$ sequential rank order strictly dominates $\mathbf{v}$, which we write $\mathbf{u}>_{S R O} \mathbf{v}$.

Important for subsequent developments is the fact that, if $\mathbf{u} \geq_{S R O} \mathbf{v}$, then $\mathbf{u}$ is a permutation of $\mathbf{v}$. Making use of (2.2), (3.4) and (3.7), we note that $\mathbf{u} \geq_{S R O} \mathbf{v}$ amounts to requiring that

$$
\begin{aligned}
& \tilde{u}_{g}^{(h)} \geqq \tilde{v}_{g}^{(h)}, \forall g=1,2, \ldots, \tilde{n}(h), \forall h=1,2, \ldots, H-1, \text { and } \\
& \tilde{u}_{g}^{(H)}=\tilde{v}_{g}^{(H)}, \forall g=1,2, \ldots, \tilde{n}(H) .
\end{aligned}
$$

We are next interested in the way utilitarian unanimity has to be adapted in order to guarantee that the corresponding ranking of heterogeneous distributions coincides with that implied by sequential rank order dominance. Before we state the result, we need to introduce the following class of $H$-tuples of functions:

$$
\boldsymbol{\Psi}:=\left\{\boldsymbol{\psi}:=\left(\psi_{1}, \ldots, \psi_{H}\right) \in \boldsymbol{\Psi}^{*} \mid \psi_{h}^{\prime}(y) \geqq \psi_{h+1}^{\prime}(y), \forall y \in \mathscr{D}, \forall h=1,2, \ldots, H-1\right\} .
$$

The connection between the class of $n$-tuples $\boldsymbol{\Psi}$ and the class of submodular functions is easily recognised if we let $g(y, h):=\psi_{h}(y)$, for all $y \in \mathscr{D}$ and all $h \in\{1,2, \ldots, n\}$. Indeed, a function $g: \mathscr{D} \times \mathscr{D} \rightarrow \mathbb{R}$ is called submodular if

$$
g(u+\Delta, v+\xi)-g(u, v+\xi) \leqq g(u+\Delta, v)-g(u, v), \forall(u, v) \in \mathscr{D} \times \mathscr{D}, \forall \Delta, \xi>0
$$

When the function $g$ is differentiable in its first argument, this reduces to the condition that

$$
g_{(1)}(y, h)=\psi_{h}^{\prime}(y) \geqq \psi_{h+1}^{\prime}(y)=g_{(1)}(y, h+1), \forall y \in \mathscr{D}, \forall h \in\{1,2, \ldots, H-1\},
$$

where $g_{(1)}(y, h)$ is the first derivative of $g(y, h)$ with respect to its first argument. Sometimes, one also says that $g$ is L-subadditive (see, e.g., Marshall and Olkin (1979, Chapter 6, Section D). Our next lemma constitutes the analogue of Lemma 2.2 when the sequential rank order criterion is used for ranking heterogeneous distributions. 
Lemma 3.2. Let $\mathbf{u}:=\left(\mathbf{u}^{1} ; \ldots ; \mathbf{u}^{H}\right), \mathbf{v}:=\left(\mathbf{v}^{1} ; \ldots ; \mathbf{v}^{H}\right) \in \mathscr{D}^{n(1)} \times \cdots \times \mathscr{D}^{n(H)}$. Then, statements (a) and (b) below are equivalent:

(a) $\sum_{h=1}^{H} \sum_{i=1}^{n(h)} \psi_{h}\left(u_{i}^{h}\right) \geqq \sum_{h=1}^{H} \sum_{i=1}^{n(h)} \psi_{h}\left(v_{i}^{h}\right)$, for all $\boldsymbol{\psi}:=\left(\psi_{1}, \ldots, \psi_{H}\right) \in \boldsymbol{\Psi}$.

(b) $\mathbf{u} \geq_{S R O} \mathbf{v}$.

\section{Proof.}

(a) $\Longrightarrow(\mathrm{b})$. Let $\boldsymbol{\lambda}:=\left(\lambda_{1}, \ldots, \lambda_{H}\right)$ and consider the $H$-tuple $\boldsymbol{\psi}:=\left(\psi_{1}, \ldots, \psi_{H}\right)$ defined by $\psi_{h}(y):=\lambda_{h} \phi(y)$, for all $h=1,2, \ldots, H$. Clearly, $\boldsymbol{\psi}:=\left(\psi_{1}, \ldots, \psi_{H}\right) \in \Psi$ provided that $\phi^{\prime}(y) \geqq 0$ and $\lambda_{1} \geqq \lambda_{2} \geqq \cdots \geqq \lambda_{H}$, which we assume. Choosing successively $\boldsymbol{\lambda}:=$ $(1,0,0, \ldots, 0,0), \boldsymbol{\lambda}:=(1,1,0, \ldots, 0,0), \ldots, \boldsymbol{\lambda}:=(1,1,1, \ldots, 1,0), \boldsymbol{\lambda}:=(1,1,1, \ldots, 1,1)$, and $\boldsymbol{\lambda}:=(-1,-1,-1, \ldots,-1,-1)$, condition (a) reduces to

$$
\begin{aligned}
& \sum_{g=1}^{n(1)} \phi\left(u_{g}^{1}\right)+\cdots+\sum_{g=1}^{n(h)} \phi\left(u_{g}^{h}\right) \geqq \sum_{g=1}^{n(1)} \phi\left(v_{g}^{1}\right)+\cdots+\sum_{g=1}^{n(h)} \phi\left(v_{g}^{h}\right), \forall h=1,2, \ldots, H-1, \text { and } \\
& \sum_{g=1}^{n(1)} \phi\left(u_{g}^{1}\right)+\cdots+\sum_{g=1}^{n(H)} \phi\left(u_{g}^{H}\right)=\sum_{g=1}^{n(1)} \phi\left(v_{g}^{1}\right)+\cdots+\sum_{g=1}^{n(h)} \phi\left(v_{g}^{H}\right),
\end{aligned}
$$

which holds for all functions $\phi \in \mathbf{\Phi}$. Appealing to Lemma 2.2, we conclude that $\mathbf{u}^{(h)} \geq_{R O} \mathbf{v}^{(h)}$, for all $h=1,2, \ldots, H-1$, and $\mathbf{u}^{(H)} \sim_{R O} \mathbf{v}^{(H)}$, hence $\mathbf{u} \geq_{S R O} \mathbf{v}$.

(b) $\Longrightarrow\left(\right.$ a). Given any $\mathbf{u}:=\left(\mathbf{u}^{1} ; \ldots ; \mathbf{u}^{H}\right) \in \mathscr{D}^{n(1)} \times \cdots \times \mathscr{D}^{n(H)}$, we have the following equality:

$$
\begin{aligned}
\sum_{h=1}^{H} \sum_{i=1}^{n(h)} \psi_{h}\left(u_{i}^{h}\right) & =\sum_{h=1}^{H}\left[\sum_{i=1}^{n(h)} \psi_{h}\left(u_{i}^{h}\right)+\sum_{k=1}^{h-1} \sum_{j=1}^{n(k)} \psi_{h}\left(u_{j}^{k}\right)-\sum_{k=1}^{h-1} \sum_{j=1}^{n(k)} \psi_{h}\left(u_{j}^{k}\right)\right] \\
& =\sum_{h=1}^{H-1}\left[\sum_{k=1}^{h} \sum_{i=1}^{n(k)}\left(\psi_{h}\left(u_{i}^{k}\right)-\psi_{h+1}\left(u_{i}^{k}\right)\right)\right]+\sum_{k=1}^{H} \sum_{i=1}^{n(k)} \psi_{H}\left(u_{i}^{k}\right) .
\end{aligned}
$$

Letting $f_{h}(y):=\psi_{h}(y)-\psi_{h+1}(y)$, for $h=1,2, \ldots, H-1$, and $f_{H}(y):=\psi_{H}(y)$, and upon substituting into (3.13), we obtain

$$
\sum_{h=1}^{H} \sum_{i=1}^{n(h)} \psi_{h}\left(u_{i}^{h}\right)=\sum_{h=1}^{H-1}\left[\sum_{k=1}^{h} \sum_{i=1}^{n(k)} f_{h}\left(u_{i}^{k}\right)\right]+\sum_{k=1}^{H} \sum_{i=1}^{n(k)} f_{H}\left(u_{i}^{k}\right)=\sum_{h=1}^{H} \sum_{i=1}^{\tilde{n}(h)} f_{h}\left(\tilde{u}_{i}^{(h)}\right) .
$$

Making use of (3.14), condition (a) can be equivalently restated as

$$
\sum_{h=1}^{H} \sum_{i=1}^{n(h)}\left(\psi_{h}\left(u_{i}^{h}\right)-\psi_{h}\left(v_{i}^{h}\right)\right)=\sum_{h=1}^{H} \sum_{i=1}^{\tilde{n}(h)}\left(f_{h}\left(\tilde{u}_{i}^{(h)}\right)-f_{h}\left(\tilde{v}_{i}^{(h)}\right)\right) \geqq 0,
$$

for all $f_{h}(y)$ that are non-decreasing in $y$. Thanks to Lemma 3.1 we can assume without loss of generality that there exists no $h \in \mathscr{H}$ and no $i \in N(h)$ such that $u_{i}^{h}=v_{i}^{h}$. Invoking the Mean Value Theorem, condition (3.15) is equivalent to

$$
\sum_{h=1}^{H-1} \sum_{i=1}^{\tilde{n}(h)} f_{h}^{\prime}\left(\xi_{i}^{h}\right)\left[\tilde{u}_{i}^{(h)}-\tilde{v}_{i}^{(h)}\right]+\sum_{i=1}^{\tilde{n}(H)} f_{H}^{\prime}\left(\xi_{i}^{H}\right)\left[\tilde{u}_{i}^{(H)}-\tilde{v}_{i}^{(H)}\right] \geqq 0,
$$


for some $\xi_{i}^{h} \in\left(\tilde{u}_{i}^{(h)}, \tilde{v}_{i}^{(h)}\right)$, for all $i \in\{1,2, \ldots, n(h)-1, n(h)\}$ and all $h \in \mathscr{H}$. By assumption we have $f_{h}^{\prime}(y)=U_{h}^{\prime}(y)-U_{h+1}^{\prime}(y) \geqq 0$, for all $y \in \mathscr{D}$ and all $h=1,2, \ldots, H-1$. We therefore conclude that it is sufficient for (3.16) to hold that $\mathbf{u}^{(h)} \geq_{R O} \mathbf{v}^{(h)}$, for all $h=1,2, \ldots, H-1$, and $\mathbf{u}^{(H)} \sim_{R O} \mathbf{v}^{(H)}$, which is precisely $\mathbf{u} \geq_{S R O} \mathbf{v}$.

A direct implication of Lemmas 3.1 and 3.2 is that the individuals who are unconcerned by the choice between two distributions - they have the same types and incomes in both situations - play no role in the ranking of these distributions by the sequential rank order criterion: removing these individuals will not affect the result of the comparisons.

As we have noted above, it follows from the very definition of the sequential rank order criterion that, if one distribution dominates another, then the dominating distribution is a permutation of the dominated one. However, not all rearrangements of incomes across individuals and types have the property that the resulting distributions constitute improvements over the initial distribution according to the sequential rank order criterion. Among the different possible rearrangements of the elements of a heterogeneous distribution, the following one will play a decisive role in our main result.

Definition 3.2. Given two heterogeneous distributions $\mathbf{u}:=\left(\mathbf{u}^{1} ; \ldots ; \mathbf{u}^{H}\right), \mathbf{v}:=\left(\mathbf{v}^{1} ; \ldots ; \mathbf{v}^{H}\right) \in$ $\mathscr{D}^{n(1)} \times \cdots \times \mathscr{D}^{n(H)}$, we say that $\mathbf{u}$ is obtained from $\mathbf{v}$ by means of a favourable permutation if there exist two types $h, k \in \mathscr{H}$ with $h<k$ and two individuals $i, j$ with $i \in N(h)$ and $j \in N(k)$ such that

$$
\begin{aligned}
& v_{i}^{h}=u_{j}^{k}<u_{i}^{h}=v_{j}^{k} ; \\
& v_{t}^{h}=u_{t}^{h}, \forall t \neq i ; \quad v_{t}^{k}=u_{t}^{k}, \forall t \neq j ; \text { and } \\
& \mathbf{u}^{(g)}=\mathbf{v}^{(g)}, \forall g \neq h, k .
\end{aligned}
$$

According to the definition above, individual $j$ is unambiguously in a better position in the heterogenous distribution $\mathbf{v}$ than individual $i$ since she has a greater income and is also ranked above by the second attribute. In other words, individual $i$ is more deprived than individual $j$ in both dimensions. It is fair to note that a favourable permutation is a particular case of what is known as a correlation increasing transformation in the literature (see, e.g., Epstein and Tanny (1980) and Tchen (1980)). To make things transparent, let us denote by $f(y, h)$ the proportion of individuals who have income $y$ and type $h$ in situation $(\mathbf{x} ; \mathbf{a}):=\left(x_{1}, \ldots, x_{n} ; a_{1}, \ldots, a_{n}\right)$. Then, Definition 3.2 amounts to requiring the existence of two income levels $u, v \in \mathscr{D}$ and two types $h, k \in \mathscr{H}$ with $u<v$ and $h<k$ such that:

$$
\begin{aligned}
& f^{*}(u, h)=f^{\circ}(u, h)-\frac{1}{n} ; f^{*}(v, h)=f^{\circ}(v, h)+\frac{1}{n} ; \\
& f^{*}(u, k)=f^{\circ}(u, k)+\frac{1}{n} ; f^{*}(v, k)=f^{\circ}(v, k)-\frac{1}{n} ; \text { and } \\
& f^{*}(s, g)=f^{\circ}(s, g), \forall(s, g) \neq(u, h),(u, k),(v, h),(v, k) ;
\end{aligned}
$$

which is nothing other than the definition of a correlation increasing transformation in the particular case where two individuals swap incomes. A favourable permutation amounts to 
compensating for the inequalities arising from the distribution of the two attributes by permuting the incomes of the rich and poor - in both attributes - individuals. Interestingly, the sequential rank order criterion has the property that a favourable permutation is always considered an improvement, as our next result demonstrates.

Lemma 3.3. Let $\mathbf{u}:=\left(\mathbf{u}^{1} ; \ldots ; \mathbf{u}^{H}\right), \mathbf{v}:=\left(\mathbf{v}^{1} ; \ldots ; \mathbf{v}^{H}\right) \in \mathscr{D}^{n(1)} \times \cdots \times \mathscr{D}^{n(H)}$. Then, $\mathbf{u} \geq_{S R O} \mathbf{v}$ whenever $\mathbf{u}$ is obtained from $\mathbf{v}$ by means of a finite sequence of favourable permutations.

Proof. Suppose that $\mathbf{u}$ is obtained from $\mathbf{v}$ by means of a single favourable permutation as defined in (3.18) so that

$$
\mathbf{u}=\left(\ldots ; v_{1}^{h}, \ldots, v_{i-1}^{h}, v_{j}^{k}, v_{i+1}^{h}, \ldots, v_{n(h)}^{h} ; \ldots ; v_{1}^{k}, \ldots, v_{j-1}^{k}, v_{i}^{h}, v_{j+1}^{k}, \ldots, v_{n(k)}^{k} ; \ldots\right)
$$

and $v_{i}^{h}<v_{j}^{k}$. Then, we have:

$$
\begin{gathered}
1 \leqq g \leqq h-1: \mathbf{u}^{(g)} \sim_{R O} \mathbf{v}^{(g)} \\
h \leqq g \leqq k-1: \mathbf{u}^{(h)}>_{R O} \mathbf{v}^{(h)} \\
k \leqq g \leqq H: \mathbf{u}^{(g)} \sim_{R O} \mathbf{v}^{(g)}
\end{gathered}
$$$$
\text { because } \mathbf{u}^{(g)}=\mathbf{v}^{(g)} \text {, }
$$$$
\text { by Lemma 2.1, }
$$

because $\mathbf{u}^{(g)}$ is a permutation of $\mathbf{v}^{(g)}$,

and we conclude that $\mathbf{u} \geq_{S R O} \mathbf{v}$. When more than one favourable permutation is needed to convert $\mathbf{u}$ into $\mathbf{v}$, the result follows by invoking the transitivity of the sequential rank order criterion.

According to Lemma 3.3, if a distribution is obtained from another distribution by means of a sequence of favourable permutations, then the former distribution sequential rank order dominates the latter. Actually, the implication also goes the other way: if one distribution is considered better than another the sequential rank order criterion, then it can be derived from the dominated distribution through an appropriate sequence of favourable permutations. Hence, our main result:

TheOREM 3.1. Let $\mathbf{u}:=\left(\mathbf{u}^{1} ; \ldots ; \mathbf{u}^{H}\right), \mathbf{v}:=\left(\mathbf{v}^{1} ; \ldots ; \mathbf{v}^{H}\right) \in \mathscr{D}^{n(1)} \times \cdots \times \mathscr{D}^{n(H)}$. Then, statements (a) and (b) below are equivalent:

(a) $\mathbf{u}$ is obtained from $\mathbf{v}$ by means of a finite sequence of favourable permutations.

(b) $\mathbf{u} \geq_{S R O} \mathbf{v}$.

Proof. Given Lemma 3.3 we only have to prove that statement (b) implies statement (a) and thanks to Lemmas 3.1 and 3.2, we can assume without loss of generality that

$$
u_{g}^{h} \neq v_{g}^{h}, \forall g \in N(h), \forall h \in \mathscr{H} .
$$

The method of proof is reminiscent of that designed for identifying the sequence of progressive transfers that allows one to recover the dominating distribution starting from the dominated distribution when one distribution is ranked above another by the Lorenz criterion (see, e.g., Berge (1963)). In the standard Lorenz case, at each step of the algorithm a progressive 
transfer is implemented with the property that in the resulting distribution there is at least one individual who is given the same income as the one she actually gets in the dominating distribution. This guarantees that, if there are $n$ individuals with distinct incomes, then it will take at most $n-1$ steps - with one progressive transfer at each step - to obtain the dominating distribution starting from the dominated one.

The general idea of our algorithm is to construct at each step a new distribution by permuting the highest income in the original distribution - that is the distribution resulting from the preceding step - with another income in such a way that:

(a) This new distribution is ranked below the dominating distribution and above the dominated one by the sequential rank order criterion; and

(b) There is at least one individual who receives in the new distribution the income she actually gets in the original one.

Unfortunately, while an appropriate choice of the individuals whose incomes are permuted ensures that the first requirement is met, it will in general take more than just one favourable permutation to satisfy the second requirement. However, after a finite number of iterations, where each iteration involves a single favourable permutation, there will be at least one individual who will be given the income she gets in the dominating distribution. This income is then removed - an operation which does not affect the ranking of the distributions - and we proceed to the next step.

Overview of the proof The algorithm that allows us to construct the dominating distribution $\mathbf{u}$ starting from the dominated distribution $\mathbf{v}$ involves $T+1$ steps as it is shown in Table 3.1 where:

$n[p]$ is the number of individuals in $\mathbf{u}[p]$ and $\mathbf{v}[p]$ whose incomes by definition differ;

$\mathbf{z}[p]$ follows from $\mathbf{v}[p]$ through a finite sequence of favourable permutations;

$\mathbf{u}[p] \geq_{S R O} \mathbf{z}[p]>_{S R O} \mathbf{v}[p]$

$u[p]_{n\left(i^{p}\right)}^{i^{p}}=z[p]_{n\left(i^{p}\right)}^{i^{p}} ;$

$\mathbf{u}[p+1]$ is obtained from $\mathbf{u}[p]$ by removing income $u[p]_{n\left(i^{p}\right)}^{i^{p}}$;

$\mathbf{v}[p+1]$ is obtained from $\mathbf{z}[p]$ by removing income $z[p]_{n\left(i^{p}\right)}^{i^{p}}$;

$n[p]-2 \leqq n[p+1] \leqq n[p]-1 ;$

for all $p=1,2, \ldots, T-1, T$. Typically, Step $p$ comprises $q^{p}$ iterations as it is described in Table 3.2. We refer the reader to Section A where a simple illustration of how the algorithm works is provided.

Detailed description of Step 1 Since the reasoning is the same for all the steps of the algorithm, we provide only the details in the case of Step 1. We remove temporarily the index 1 to simplify notation and we therefore use $\mathbf{u}$ and $\mathbf{v}$ in place of $\mathbf{u}[1]$ and $\mathbf{v}[1]$, respectively. 


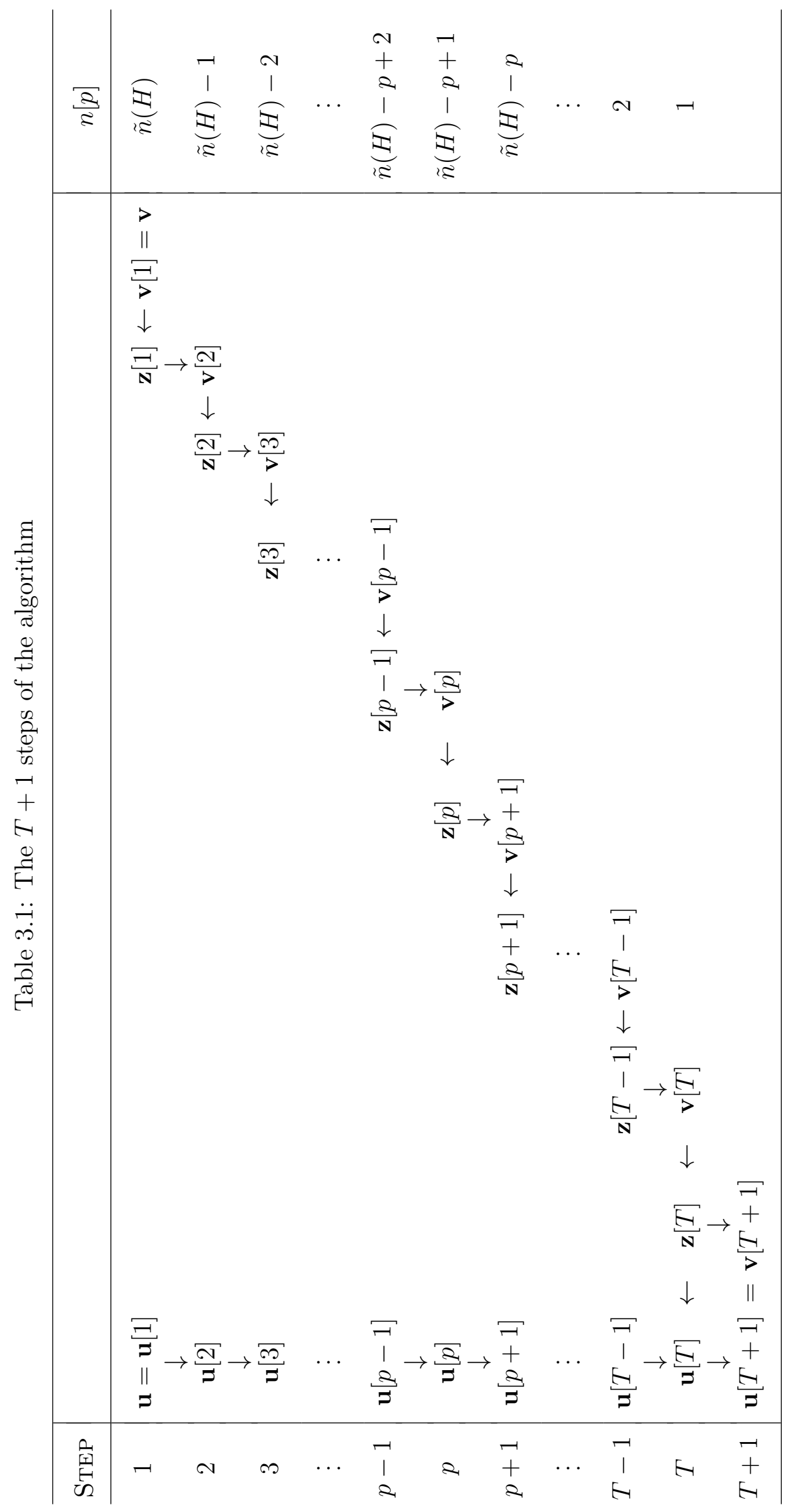




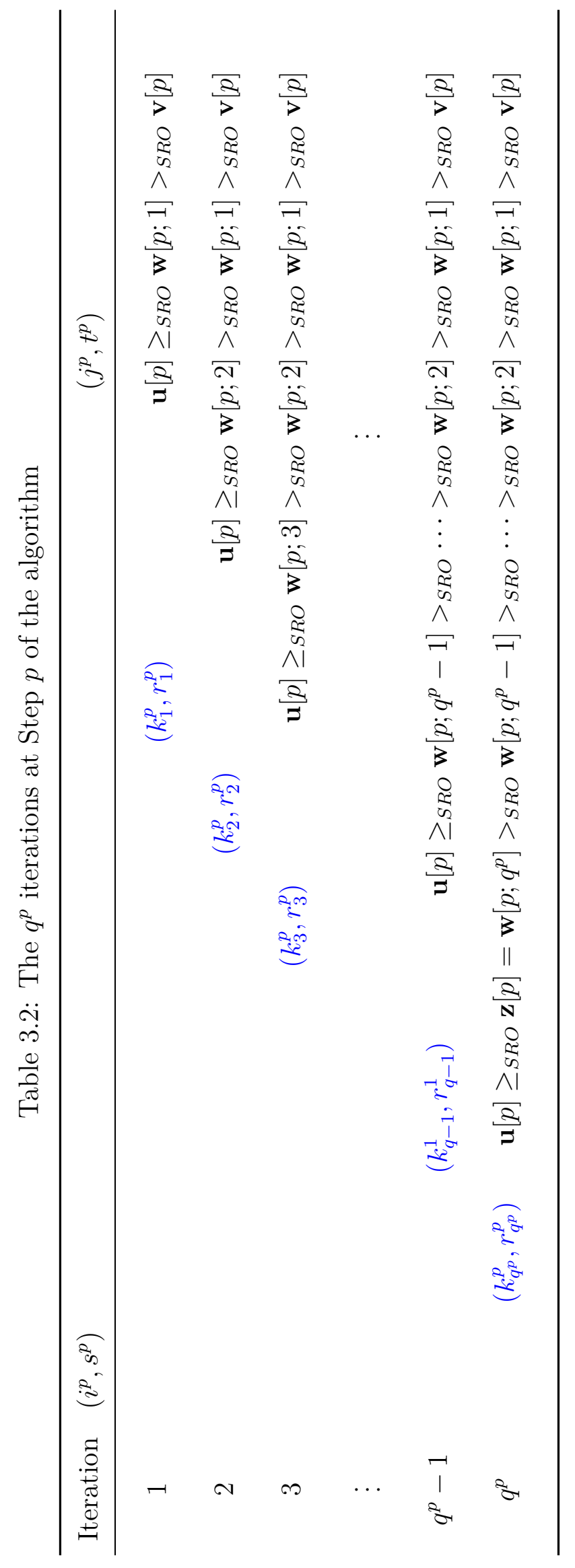


1.A. At the beginning of Step 1, we identify those two individuals who in distributions $\mathbf{u}$ and $\mathbf{v}$ receive the maximum available income. We verify that the individual who gets the maximum income in distribution $\mathbf{u}$ has a lower type than the individual who is given the same income in distribution $\mathbf{v}$. Consider the four following indices:

$$
\begin{aligned}
& i:=\min \left\{h \in \mathscr{H} \mid \exists g \in N(h): u_{g}^{h} \geqq u_{\ell}^{p}, \forall \ell \in N(p), \forall p \in \mathscr{H}\right\} ; \\
& j:=\min \left\{h \in \mathscr{H} \mid \exists g \in N(h): v_{g}^{h} \geqq v_{\ell}^{p}, \forall \ell \in N(p), \forall p \in \mathscr{H}\right\} ; \\
& s:=\min \left\{g \in N(i) \mid u_{g}^{i} \geqq u_{\ell}^{i}, \forall \ell \in N(i)\right\} ; \\
& t:=\min \left\{g \in N(j) \mid v_{g}^{j} \geqq v_{\ell}^{j}, \forall \ell \in N(j)\right\} .
\end{aligned}
$$

By definition of the indices $i, j, s$ and $t$, we have

$$
\begin{aligned}
& u_{1}^{i} \leqq \cdots \leqq u_{s-1}^{i}<u_{s}^{i}=u_{s+1}^{i}=\cdots=u_{n(i)}^{i} ; \\
& u_{g}^{h}<u_{s}^{i}, \forall g \in N(h), \forall h=1,2, \ldots, i-1 ; \\
& u_{s}^{i} \geqq u_{g}^{h}, \forall g \in N(h), \forall h=i+1, i+2, \ldots, H ; \\
& v_{1}^{j} \leqq \cdots \leqq v_{t-1}^{j}<v_{t}^{j}=v_{t+1}^{j}=\cdots=v_{n(j)}^{j} ; \\
& v_{g}^{h}<v_{t}^{j}, \forall g \in N(h), \forall h=1,2, \ldots, j-1 ; \\
& v_{t}^{j} \geqq v_{g}^{h}, \forall g \in N(h), \forall h=j+1, i+2, \ldots, H .
\end{aligned}
$$

Furthermore, $u_{s}^{i}:=\max \left\{u_{g}^{h}\right\}=\max \left\{v_{g}^{h}\right\}=: v_{t}^{j}$, because $\mathbf{u}$ is a permutation of $\mathbf{v}$. We note that by construction $i<j$. Indeed, we cannot have $i=j$, for, if it were the case, then we would have

$$
u_{n(i)}^{i}=v_{n(i)}^{i},
$$

which is ruled out by (3.21). Suppose next that $i>j$ and consider the distributions $\mathbf{u}^{(j)}:=$ $\left(\mathbf{u}^{1} ; \mathbf{u}^{2} ; \ldots ; \mathbf{u}^{j}\right)$ and $\mathbf{v}^{(j)}:=\left(\mathbf{v}^{1} ; \mathbf{v}^{2} ; \ldots ; \mathbf{v}^{j}\right)$. By the definition of $v_{t}^{j}$, we have

$$
\tilde{u}_{\tilde{n}(j)}^{(j)}<u_{s}^{i}=v_{t}^{j}=\tilde{v}_{\tilde{n}(j)}^{(j)} .
$$

Hence $\neg\left[\mathbf{u}^{(j)} \geq_{R O} \mathbf{v}^{(j)}\right]$, which contradicts the fact that $\mathbf{u} \geq_{S R O} \mathbf{v}$ by assumption. We therefore have the structure depicted in Table 3.3. Now we operate favourable permutations within the subpopulation $N(i) \cup N(i+1) \cup \cdots \cup N(j-1) \cup N(j)$ in such a way that, at the end of this process, one obtains a distribution $\mathbf{z}[1]$ such that (i) $u_{n(i)}^{i}=z[1]_{n(i)}^{i}=v_{t}^{j}$ and (ii) $\mathbf{u} \geq_{S R O} \mathbf{z}[1]>_{S R O} \mathbf{v}$.

1.B. We look for the type $k \in\{i, i+1, \ldots, j-1\}$ such that one of its members $r$ has the largest income in the distribution $\left(\mathbf{v}^{i} ; \mathbf{v}^{i+1} ; \ldots ; \mathbf{v}^{j-2} ; \mathbf{v}^{j-1}\right)$. Since by definition the income $v_{r}^{k}$ is smaller than $v_{t}^{j}$ it is possible to perform a favourable permutation involving the individual $r \in N(k)$ and the individual $t \in N(j)$. Let

(3.26a) $k:=\min \left\{h \in\{i, \ldots, j-1\} \mid \exists g \in N(h): v_{g}^{h} \geqq v_{\ell}^{p}, \forall \ell \in N(p), \forall p \in\{i, \ldots, j-1\}\right\}$;

(3.26b) $r:=\min \left\{g \in N(k) \mid v_{g}^{k} \geqq v_{\ell}^{k}, \forall \ell \in N(k)\right\}$. 
Table 3.3: Selection of the couples $(i, s)$ and $(j, t)$ at the initialisation stage

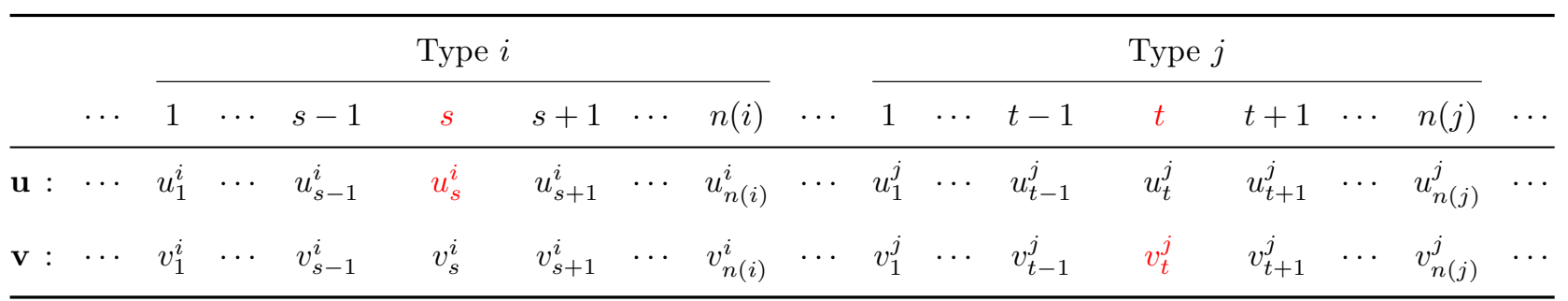

We note that it is possible that $k=i$ and therefore that $r \in\{1,2, \ldots, s-1\}$. By definition of the indices $k$ and $r$, we have:

$$
\begin{aligned}
& v_{r}^{k} \leqq \cdots \leqq v_{r-1}^{k} \leqq v_{r}^{k}=v_{r+1}^{k}=\cdots=v_{n(k)}^{k} ; \\
& v_{g}^{h}<v_{r}^{k}, \forall g \in N(h), \forall h \in\{i, i+1, \ldots, k-1\} ; \\
& v_{r}^{k} \geqq v_{g}^{h}, \forall g \in N(h), \forall h \in\{k+1, k+2, \ldots, j-1\} ; \\
& v_{r}^{k}<v_{t}^{j} .
\end{aligned}
$$

Consider next the distribution $\mathbf{w}:=\left(\mathbf{w}^{1} ; \ldots ; \mathbf{w}^{H}\right)$ obtained from $\mathbf{v}:=\left(\mathbf{v}^{1} ; \ldots ; \mathbf{v}^{H}\right)$ by means of a favourable permutation as is indicated below:

$$
\begin{aligned}
& \mathbf{w}^{h}=\mathbf{v}^{h}, \forall h \in \mathscr{H}(h \neq k, j) ; \\
& w_{g}^{k}=v_{g}^{k}, \forall g \in N(k)(g \neq n(k)) ; \\
& w_{n(k)}^{k}=v_{t}^{j} ; \\
& w_{g}^{j}=v_{g}^{j}, \forall g \in\{1,2, \ldots, p-1\} \cup\{t+1, t+2, \ldots, n(j)\} ; \\
& w_{t}^{j}=v_{n(k)}^{k}=v_{r}^{k} ; \\
& w_{g}^{j}=v_{g-1}^{j}, \forall g \in\{p+1, p+2, \ldots, t-1, t\} ;
\end{aligned}
$$

\begin{tabular}{|c|c|c|c|c|c|c|c|c|c|c|c|c|c|c|c|c|}
\hline & \multicolumn{4}{|c|}{ Type $i$} & \multicolumn{6}{|c|}{ Type $k$} & \multirow[b]{2}{*}{$\cdots$} & \multicolumn{5}{|c|}{ Type $j$} \\
\hline$\cdots$ & 1 & $\cdots$ & $s$ & $n(i)$ & $\cdots$ & 1 & $\cdots$ & $r$ & $\cdots$ & $n(k)$ & & 1 & $\cdots$ & $p$ & $\cdots$ & $\cdots \quad n(j)$ \\
\hline $\mathbf{u}: \cdots$ & $u_{1}^{i}$ & & $u_{s}^{i}$ & $u_{n(i)}^{i}$ & $\cdots$ & $u_{1}^{k}$ & $\cdots$ & $u_{r}^{k}$ & & $u_{n(k)}^{k}$ & $\cdots$ & $u_{1}^{j}$ & $\cdots$ & $u_{p}^{j}$ & $\cdots$ & $\cdots \quad u_{n(j)}^{j}$ \\
\hline $\mathbf{w}: \cdots$ & $v_{1}^{i}$ & & $v_{s}^{i}$ & $v_{n(i)}^{i}$ & & $v_{1}^{k}$ & $\ldots$ & $v_{r}^{k}$ & & $v_{t}^{j}$ & $\cdots$ & $v_{1}^{j}$ & & $v_{n(k)}^{k}$ & $\begin{array}{ll}\cdots & v_{t-1}^{j}\end{array}$ & $\cdots \quad v_{n(j)}^{j}$ \\
\hline $\mathbf{v}: \cdots$ & $v_{1}^{i}$ & & $v_{s}^{i}$ & $v_{n(i)}^{i}$ & & $v_{1}^{k}$ & & $v_{r}^{k}$ & & $v_{n(k)}^{k}$ & $\cdots$ & $v_{1}^{j}$ & & $v_{p}^{j}$ & $\ldots$ & $\cdots \quad v_{n(j)}^{j}$ \\
\hline
\end{tabular}

where the index $p$ is defined by

$$
v_{1}^{j} \leqq \cdots \leqq v_{p-1}^{j} \leqq v_{r}^{k}<v_{p}^{j} \leqq v_{p+1}^{j} \leqq \cdots \leqq v_{t-1}^{j}<v_{t}^{j}=v_{t+1}^{j}=\cdots=v_{n(j)}^{j} .
$$

The construction of distribution $\mathbf{w}:=\left(\mathbf{w}^{1} ; \ldots ; \mathbf{w}^{H}\right)$ is illustrated in Table 3.4. It is important

Table 3.4: Construction of $\mathbf{w}$ starting from $\mathbf{v}$ by means of a favourable permutation

to bear in mind the fact that it is individual $n(k)$ of type $k$ who is exchanging her income 
with individual $t$ of type $j$ in order to make sure that the positions of the individuals of types $k$ and $j$ are not affected. Note also that the incomes of the individuals of type $j$ in the distribution $\mathbf{w}$ have been rearranged in non-decreasing order to fit the definition of a heterogeneous distribution.

1.C. Since distribution $\mathbf{w}$ is obtained from distribution $\mathbf{v}$ by means of a favourable permutation, it follows from Lemma 3.2 that $\mathbf{w}>_{S R O} \mathbf{v}$. It remains to be verified that $\mathbf{u} \geq_{S R O} \mathbf{w}$ and, to this end, we examine the distributions $\mathbf{u}^{(h)}, \mathbf{w}^{(h)}$ and $\mathbf{v}^{(h)}$, for $h=1,2, \ldots, H$. We consider successively four cases.

CASE 1: $1 \leqq h \leqq i-1$. By assumption $\mathbf{u}^{(h)} \geq_{R O} \mathbf{v}^{(h)}$ and by construction $\mathbf{w}^{(h)}=\mathbf{v}^{(h)}$. Hence, $\tilde{u}_{g}^{(h)} \geqq \tilde{w}_{g}^{(h)}=\tilde{v}_{g}^{(h)}$, for all $g \in N(h)$.

CASE 2: $i \leqq h \leqq j-1$. If $k \in h+1, h+2, \ldots, j-1$, then the argument is the same as in Case 1 above. Therefore, we restrict attention to the case in which $k \in\{i, i+1, \ldots, h-1, h\}$. We indicate by $g^{*}$ the highest rank with the income $v_{r}^{k}$ in the ordered distribution $\tilde{\mathbf{v}}^{(h)}$, as shown in

$$
\tilde{v}_{1}^{(h)} \leqq \cdots \leqq \tilde{v}_{g^{*}-(n(k)-r)-1}^{(h)} \leqq \tilde{v}_{g^{*}-(n(k)-r)}^{(h)}=\cdots=\tilde{v}_{g^{*}}^{(h)} \equiv v_{n(k)}^{k}<\tilde{v}_{g^{*}+1}^{(h)} \leqq \cdots \leqq \tilde{v}_{\tilde{n}(h)}^{(h)},
$$

where we have made use of the fact that by definition $v_{r}^{k}=v_{g}^{k}$, for all $g=r+1, r+2, \ldots, n(k)$. We indicate by

$$
\rho^{*}:=\#\left\{g \in\{1,2, \ldots, \tilde{n}(h)-1, \tilde{n}(h)\} \mid \tilde{v}_{g}^{(h)}>\tilde{v}_{g^{*}}^{(h)}=v_{r}^{k}\right\}=\tilde{n}(h)-g^{*}
$$

the number of individuals who in the distribution $\mathbf{v}^{(h)}:=\left(\mathbf{v}^{1} ; \mathbf{v}^{2} ; \ldots ; \mathbf{v}^{h}\right)$ have incomes greater than $v_{r}^{k}$. Because by definition $v_{r}^{k} \geqq v_{g}^{h}$, for all $g \in N(h)$ and all $h \in\{i, i+1, \ldots, j-1\}$, we note that

$$
\tilde{n}(h)-\tilde{n}(i-1) \leqq g^{*} \leqq \tilde{n}(h)
$$

or equivalently that

$$
\rho^{*} \leqq \tilde{n}(h)-(n(i)+n(i+1)+\cdots+n(h))=\tilde{n}(i-1) .
$$

Subcase 2.1: $g^{*}=\tilde{n}(h)$. Then, it follows from the definition of $\tilde{\mathbf{w}}^{(h)}$ and the fact that $\tilde{\mathbf{u}}^{(h)} \geq_{R O} \tilde{\mathbf{v}}^{(h)}$ by assumption that

$$
\begin{aligned}
& \tilde{u}_{g}^{(h)} \geqq \tilde{w}_{g}^{(h)}=\tilde{v}_{g}^{(h)}, \forall g=1,2, \ldots, \tilde{n}(h)-1, \text { and } \\
& u_{s}^{i}=\tilde{u}_{\tilde{n}(h)}^{(h)}=\tilde{w}_{\tilde{n}(h)}^{(h)}=v_{t}^{j}>v_{r}^{k}=v_{n(k)}^{k} \equiv \tilde{v}_{\tilde{n}(h)}^{(h)} .
\end{aligned}
$$

SUbCASE 2.2: $g^{*} \in\{1,2, \ldots, \tilde{n}(h)-1\}$. Invoking again the definition of $\tilde{\mathbf{w}}^{(h)}$ and the fact that $\tilde{\mathbf{u}}^{(h)} \geq_{R O} \tilde{\mathbf{v}}^{(h)}$ by assumption, we have

$$
\begin{aligned}
& \tilde{u}_{g}^{(h)} \geqq \tilde{w}_{g}^{(h)}=\tilde{v}_{g}^{(h)}, \forall g=1,2, \ldots, g^{*}-1, \text { and } \\
& u_{s}^{i}=\tilde{u}_{\tilde{n}(h)}^{(h)}=\tilde{w}_{\tilde{n}(h)}^{(h)}=v_{t}^{j}>v_{r}^{k}=v_{n(k)}^{k} \equiv \tilde{v}_{\tilde{n}(h)}^{(h)} .
\end{aligned}
$$


It remains to be seen what happens when $g \in\left\{g^{*}, g^{*}+1, \ldots, \tilde{n}(h)-1\right\}$.

Starting With The Distribution $\tilde{u}^{(h)}$ and by definition of the indices $i$ and $s$, we have

$$
\tilde{u}_{1}^{(h)} \leqq \cdots \leqq \tilde{u}_{\tilde{n}(h-1)+1}^{(h)} \leqq \cdots \leqq \tilde{u}_{\tilde{n}(h-1)+s-1}^{(h)}<\tilde{u}_{\tilde{n}(h-1)+s}^{(h)}=\cdots=\tilde{u}_{\tilde{n}(h)}^{(h)}=u_{s}^{i} .
$$

Given a non-empty and finite set $A:=\left\{a_{1}, a_{2}, \ldots, a_{m}\right\}$, where $a_{i} \in \mathbb{R}$, for all $i=1,2, \ldots, m$ $(m \geqq 2)$, we denote by $\max _{\rho} A$ the $\rho^{\text {th }}$-greatest element in $A$ with $1 \leqq \rho \leqq m$. Since by definition

$$
\tilde{u}_{\tilde{n}(h)}^{(h)}=u_{s}^{i}>\tilde{u}_{g}^{(i-1)}, \forall g=1,2, \ldots, \tilde{n}(i-1)-1, \tilde{n}(i-1),
$$

we deduce that

$$
\left\{\left\{\tilde{u}_{g}^{(i-1)}\right\}_{g=1,2, \ldots, \tilde{n}(i-1)}\right\} \subseteq\left\{\left\{\tilde{u}_{g}^{(h)}\right\}_{g=1,2, \ldots, \tilde{n}(h)-1}\right\}
$$

Thus we have:

$$
\begin{aligned}
& \tilde{u}_{\tilde{n}(h)-1}^{(h)}=\max _{1}\left\{\left\{u_{g}^{(h)}\right\}_{g=1,2, \ldots, \tilde{n}(h)-1}\right\} \geqq \max _{1}\left\{\left\{u_{g}^{(i-1)}\right\}_{g=1,2, \ldots, \tilde{n}(i-1)}\right\}=\tilde{u}_{\tilde{n}(i-1)-0}^{(i-1)} ; \quad \rho=1 \\
& \tilde{u}_{\tilde{n}(h)-2}^{(h)}=\max _{2}\left\{\left\{u_{g}^{(h)}\right\}_{g=1,2, \ldots, \tilde{n}(h)-1}\right\} \geqq \max _{2}\left\{\left\{u_{g}^{(i-1)}\right\}_{g=1,2, \ldots, \tilde{n}(i-1)}\right\}=\tilde{u}_{\tilde{n}(i-1)-1}^{(i-1)} ; \quad \rho=2 \\
& \tilde{u}_{\tilde{n}(h)-\rho^{*}+1}^{(h)}=\max _{\rho^{*}-1}\left\{\left\{u_{g}^{(h)}\right\}_{g=1,2, \ldots, \tilde{n}(h)-1}\right\} \geqq \max _{\rho^{*}-1}\left\{\left\{u_{g}^{(i-1)}\right\}_{g=1,2, \ldots, \tilde{n}(i-1)}\right\}=\tilde{u}_{\tilde{n}(i-1)-\rho^{*}+2}^{(i-1)} ; \rho=\rho^{*}-1 \\
& \tilde{u}_{\tilde{n}(h)-\rho^{*}}^{(h)}=\max _{\rho^{*}}\left\{\left\{u_{g}^{(h)}\right\}_{g=1,2, \ldots, \tilde{n}(h)-1}\right\} \geqq \max _{\rho^{*}}\left\{\left\{u_{g}^{(i-1)}\right\}_{g=1,2, \ldots, \tilde{n}(i-1)}\right\}=\tilde{u}_{\tilde{n}(i-1)-\rho^{*}+1}^{(i-1)} ; \quad \rho=\rho^{*} .
\end{aligned}
$$

More compactly:

$$
\tilde{u}_{(\tilde{n}(h)-1)-\rho}^{(h)}=\max _{\rho}\left\{\left\{u_{g}^{(h)}\right\}_{g=1,2, \ldots, \tilde{n}(h)-1}\right\} \geqq \max _{\rho}\left\{\left\{u_{g}^{(i-1)}\right\}_{g=1,2, \ldots, \tilde{n}(i-1)}\right\}=\tilde{u}_{\tilde{n}(i-1)-\rho+1}^{(i-1)},
$$

for all $\rho=1,2, \ldots, \rho^{*}-1, \rho^{*}$.

Starting With The Distribution $\tilde{v}^{(h)}$ and since by definition $\tilde{v}_{g^{*}}^{(h)}=v_{n(k)}^{k} \geqq v_{g}^{p}$, for all $g \in N(p)$ and all $p=i, i+1, \ldots, h$, we deduce from (3.31) that

$$
\left\{\left\{\tilde{v}_{g}^{(h)}\right\}_{g=g^{*}+1, g^{*}+2, \ldots, \tilde{n}(h)}\right\} \subseteq\left\{\left\{\tilde{v}_{g}^{(i-1)}\right\}_{g=1,2, \ldots, \tilde{n}(i-1)}\right\} .
$$

This implies in turn that

$$
\begin{gathered}
\tilde{v}_{\tilde{n}(h)-0}^{(h)}=\max _{1}\left\{\left\{\tilde{v}_{g}^{(h)}\right\}_{g=g^{*}+1, \ldots, \tilde{n}(h)}\right\} \leqq \max _{1}\left\{\left\{\tilde{v}_{g}^{(i-1)}\right\}_{g=1,2, \ldots, \tilde{n}(i-1)}\right\}=\tilde{v}_{\tilde{n}(i-1)-0}^{(i-1)} ; \\
\tilde{v}_{\tilde{n}(h)-1}^{(h)}=\max _{2}\left\{\left\{\tilde{v}_{g}^{(h)}\right\}_{g=g^{*}+1, \ldots, \tilde{n}(h)}\right\} \leqq \max _{2}\left\{\left\{\tilde{v}_{g}^{(i-1)}\right\}_{g=1,2, \ldots, \tilde{n}(i-1)}\right\}=\tilde{v}_{\tilde{n}(i-1)-1}^{(i-1)} ; \\
\vdots \\
\tilde{v}_{\tilde{n}(h)-\rho^{*}+2}^{(h)}=\max _{\rho^{*}-1}\left\{\left\{\tilde{v}_{g}^{(h)}\right\}_{g=g^{*}+1, \ldots, \tilde{n}(h)}\right\} \leqq \max _{\rho^{*}-1}\left\{\left\{\tilde{v}_{g}^{(i-1)}\right\}_{g=1,2, \ldots, \tilde{n}(i-1)}\right\}=\tilde{v}_{\tilde{n}(i-1)-\rho^{*}+2}^{(i-1)} ; \rho=\rho^{*}-1 \\
\tilde{v}_{\tilde{n}(h)-\rho^{*}+1}^{(h)}=\max _{\rho^{*}}\left\{\left\{\tilde{v}_{g}^{(h)}\right\}_{g=g^{*}+1, \ldots, \tilde{n}(h)}\right\} \leqq \max _{\rho^{*}}\left\{\left\{\tilde{v}_{g}^{(i-1)}\right\}_{g=1,2, \ldots, \tilde{n}(i-1)}\right\}=\tilde{v}_{\tilde{n}(i-1)-\rho^{*}+1}^{(i-1)} ; \quad \rho=\rho^{*} .
\end{gathered}
$$


More compactly:

$$
\tilde{v}_{\tilde{n}(h)-\rho+1}^{(h)}=\max _{\rho}\left\{\left\{\tilde{v}_{g}^{(h)}\right\}_{g=g^{*}+1, g^{*}+2, \ldots, \tilde{n}(h)}\right\} \leqq \max _{\rho}\left\{\left\{\tilde{v}_{g}^{(i-1)}\right\}_{g=1,2, \ldots, \tilde{n}(i-1)}\right\}=\tilde{v}_{\tilde{n}(i-1)-\rho+1}^{(i-1)},
$$

for all $\rho=1,2, \ldots, \rho^{*}-1, \rho^{*}$.

Combining (3.39) AND (3.41) and making use of the definition of $\mathbf{w}$ and of the fact that $\mathbf{u}^{(i-1)} \geq_{R O} \mathbf{v}^{(i-1)}$, we obtain

$$
\begin{array}{cr}
\tilde{u}_{\tilde{n}(h)-1}^{(h)} \geqq \tilde{u}_{\tilde{n}(i-1)-0}^{(i-1)} \geqq \tilde{v}_{\tilde{n}(i-1)-0}^{(i-1)} \geqq \tilde{v}_{\tilde{n}(h)-0}^{(h)}=\tilde{w}_{\tilde{n}(h)-1}^{(h)} \geqq \tilde{v}_{\tilde{n}(h)-1}^{(h)} ; & \rho=1 \\
\tilde{u}_{\tilde{n}(h)-2}^{(h)} \geqq \tilde{u}_{\tilde{n}(i-1)-1}^{(i-1)} \geqq \tilde{v}_{\tilde{n}(i-1)-1}^{(i-1)} \geqq \tilde{v}_{\tilde{n}(h)-1}^{(h)}=\tilde{w}_{\tilde{n}(h)-2}^{(h)} \geqq \tilde{v}_{\tilde{n}(h)-2}^{(h)} ; & \rho=2 \\
\vdots & \vdots \\
\tilde{u}_{\tilde{n}(h)-\rho^{*}+1}^{(h)} \geqq \tilde{u}_{\tilde{n}(i-1)-\rho^{*}+2}^{(i-1)} \geqq \tilde{v}_{\tilde{n}(i-1)-\rho^{*}+2}^{(i-1)} \geqq \tilde{v}_{\tilde{n}(h)-\rho^{*}+2}^{(h)}=\tilde{w}_{\tilde{n}(h)-\rho^{*}+1}^{(h)} \geqq \tilde{v}_{\tilde{n}(h)-\rho^{*}+1}^{(h)} ; \quad \rho=\rho^{*}-1 \\
\tilde{u}_{\tilde{n}(h)-\rho^{*}}^{(h)} \geqq \tilde{u}_{\tilde{n}(i-1)-\rho^{*}+1}^{(i-1)} \geqq \tilde{v}_{\tilde{n}(i-1)-\rho^{*}+1}^{(i-1)} \geqq \tilde{v}_{\tilde{n}(h)-\rho^{*}+1}^{(h)}=\tilde{w}_{\tilde{n}(h)-\rho^{*}}^{(h)} \geqq \tilde{v}_{\tilde{n}(h)-\rho^{*}}^{(h)} ; & \rho=\rho^{*} .
\end{array}
$$

More compactly:

$$
\tilde{u}_{\tilde{n}(h)-\rho}^{(h)} \geqq \tilde{u}_{\tilde{n}(i-1)-\rho+1}^{(i-1)} \geqq \tilde{v}_{\tilde{n}(i-1)-\rho+1}^{(i-1)} \geqq \tilde{v}_{\tilde{n}(h)-\rho+1}^{(h)}=\tilde{w}_{\tilde{n}(h)-\rho}^{(h)} \geqq \tilde{v}_{\tilde{n}(h)-\rho}^{(h)},
$$

for all $\rho=1,2, \ldots, \rho^{*}-1, \rho^{*}$, or equivalently

$$
\tilde{u}_{g}^{(h)} \geqq \tilde{u}_{g+1}^{(i-1)} \geqq \tilde{v}_{g+1}^{(i-1)} \geqq \tilde{v}_{g+1}^{(h)}=\tilde{w}_{g}^{(h)} \geqq \tilde{v}_{g}^{(h)},
$$

for all $g=g^{*}, g^{*}+1, \ldots, \tilde{n}(h)-2, \tilde{n}(h)-1$. Thus, we conclude that

$$
\tilde{u}_{g}^{(h)} \geqq \tilde{w}_{g}^{(h)} \geqq \tilde{v}_{g}^{(h)}, \forall g=g^{*}, g^{*}+1, \ldots, \tilde{n}(h)-2, \tilde{n}(h)-1 .
$$

Finally, collecting equations (3.34a), (3.34b) on the one hand, and equations (3.35a), (3.35b) and (3.44) on the other, we obtain $\tilde{u}_{g}^{(h)} \geqq \tilde{w}_{g}^{(h)} \geqq \tilde{v}_{g}^{(h)}$, for all $h \in\{i, i+1, \ldots, j-1\}$.

CASE 3: $j \leqq h \leqq H-1$. By assumption $\mathbf{u}^{(h)} \geq_{R O} \mathbf{v}^{(h)}$ and by construction $\mathbf{w}^{(h)}$ is a permutation of $\mathbf{v}^{(h)}$. Hence, $\tilde{u}_{g}^{(h)} \geqq \tilde{w}_{g}^{(h)}=\tilde{v}_{g}^{(h)}$, for all $g \in N(h)$.

CASE $4: h=H$. By assumption $\mathbf{u}^{(H)} \sim_{R O} \mathbf{v}^{(H)}$ and by construction $\mathbf{w}^{(H)}$ is a permutation of $\mathbf{v}^{(H)}$. Hence, $\tilde{u}_{g}^{(H)}=\tilde{w}_{g}^{(H)}=\tilde{v}_{g}^{(H)}$, for all $g \in N(H)$.

To summarise, we have $\mathbf{u}^{(h)} \geq_{R O} \mathbf{w}^{(h)} \geq_{R O} \mathbf{v}^{(h)}$, for all $h=1,2, \ldots, H-1$, with $\mathbf{w}^{(h)}>_{R O} \mathbf{v}^{(h)}$, for at least one $h$, and $\mathbf{u}^{(H)} \sim_{R O} \mathbf{w}^{(H)} \sim_{R O} \mathbf{v}^{(H)}$, hence $\mathbf{u} \geq_{S R O} \mathbf{w}>_{S R O} \mathbf{v}$.

To complete the argument In what follows we reintroduce the subscripts and superscripts in order to identify the step and the iteration we refer to. To this end, we use $i^{1}, j^{1}, s^{1}$, and $t^{1}$ in place of $i, j, s$, and $t$, respectively, where the superscript "1" refers to Step 1. Similarly, we write $k_{1}^{1}$ and $r_{1}^{1}$ in place of $k$ and $r$, where the superscript "1" refers to Step 1 and the subscript "1" to the first iteration of Step 1. Finally, we substitute $\mathbf{w}[1,1]$ for $\mathbf{w}$ to make clear that it is the distribution we have arrived at after the first iteration of Step 1. Given these conventions, we have $\mathbf{u}[1] \geq_{S R O} \mathbf{w}[1,1]>_{S R O} \mathbf{v}[1]$ and there are two possibilities. 
CASE 1: $w[1,1]_{n\left(i^{1}\right)}^{i^{1}}=u[1]_{n\left(i^{1}\right)}^{i^{1}}$, which occurs if $k_{1}^{1}=i^{1}$. Then, we let $\mathbf{z}[1]=\mathbf{w}[1,1]$ and we remove individual $n\left(i^{1}\right)$ of type $i^{1}$ from the distributions $\mathbf{u}[1]$ and $\mathbf{z}[1]$ to obtain the distributions $\mathbf{u}[2]$ and $\mathbf{v}[2]$, respectively, which differ component by component. Invoking Lemma 3.3, we have:

$$
\mathbf{u}[1] \sim_{S R O} \mathbf{u}[2] \geq_{S R O} \mathbf{v}[2] \sim_{S R O} \mathbf{z}[1]=\mathbf{w}[1,1]>_{S R O} \mathbf{v}[1]
$$

and we proceed to Step 2.

CASE 2: $w[1,1]_{n\left(i^{1}\right)}^{i^{1}}<u[1]_{n\left(i^{1}\right)}^{i^{1}}$, which occurs if $k_{1}^{1}>i^{1}$. We apply the above reasoning to the distributions $\mathbf{u}[1]$ and $\mathbf{w}[1,1]$ keeping in mind that by definition $k_{1}^{1}<j^{1}$. By so doing, we select a type $k_{2}^{1}<k_{1}^{1}$ such that one of its members $r_{2}^{1} \in N\left(k_{2}^{1}\right)$ has the largest income in the distribution $\left(\mathbf{w}[1,1]^{i} ; \mathbf{w}[1,1]^{i+1} ; \ldots ; \mathbf{w}[1,1]^{k_{1}^{1}-2} ; \mathbf{w}[1,1]^{k_{1}^{1}-1}\right)$. By permuting the incomes of individuals $r_{2}^{1} \in N\left(k_{2}^{1}\right)$ and $r_{1}^{1} \in N\left(k_{1}^{1}\right)$ in distribution $\mathbf{w}[1,1]$ we construct the distribution $\mathbf{w}[1,2]$ which has the property that

$$
\mathbf{u}[1] \geq_{S R O} \mathbf{w}[1,2]>_{S R O} \mathbf{w}[1,1]>_{S R O} \mathbf{v}[1]
$$

We face again two possibilities - either $k_{2}^{1}=i^{1}$, or $k_{2}^{1}>i^{1}$ - and we proceed as above. Repeating this argument, we obtain after a finite number of $q^{1}$ iterations a distribution $\mathbf{w}\left[1, q^{1}\right]$ such that

$$
\begin{aligned}
& \mathbf{u}[1] \geq_{S R O} \mathbf{w}\left[1, q^{1}\right]>_{S R O} \mathbf{w}\left[1, q^{1}-1\right]>_{S R O} \cdots>_{S R O} \mathbf{w}[1,1]>_{S R O} \mathbf{v}[1] \text { and } \\
& w\left[1, q^{1}\right]_{n\left(i^{1}\right)}^{i^{1}}=u[1]_{n\left(i^{1}\right)}^{i^{1}} .
\end{aligned}
$$

where $q^{1} \leqq j^{1}-i^{1} \leqq H-1$ (see Table 3.1 ). We are in the situation described in Case 1 above and we can proceed to Step 2.

In the worst case where $j^{p}=H$ and $i^{p}=1$, it will take $(H-1)$ iterations at Step $p$ to obtain a distribution $\mathbf{w}\left[p, q^{p}\right]$ verifying

$$
\begin{aligned}
& \mathbf{u}[p] \geq_{S R O} \mathbf{w}\left[p, q^{p}\right]>_{S R O} \mathbf{w}\left[p, q^{p}-1\right]>_{S R O} \cdots>_{S R O} \mathbf{w}[p, 1]>_{S R O} \mathbf{v}[p] \text { and } \\
& w\left[p, q^{p}\right]_{n\left(k_{q}^{p}\right)}^{k_{q}^{p}}=u_{n\left(i^{p}\right)}^{i^{p}} .
\end{aligned}
$$

Since at the end of each step one income at least is removed from the distributions under comparison, we conclude that it takes at most $\tilde{n}(H)$ steps to obtain distribution $\mathbf{u}$ from distribution $\mathbf{v}$ by means of favourable permutations, where each step comprises no more than $(H-1)$ iterations.

\section{Favourable Permutations and Inequality Reduction}

Favourable permutations and inequality of well-being No doubt the main reason why the notion of favourable permutations have met a great success in the economics of multidimensional welfare and inequality measurement is because it is related to the idea of compensation introduced by Roemer (1996) or Fleurbaey (2008) among others. Typically, the idea is to 
treat the attributes entering the utility function in an asymmetrical way by assuming that one attribute is used to compensate another (see, e.g., Muller and Trannoy (2012)). A favourable permutation amounts to compensating for the inequalities arising from the distribution of the two attributes by permuting the incomes of the rich and poor - in both attributes - individuals. Put differently, the permutation of the endowments in the cardinal attribute reduces the pairwise correlation - or positive association - existing between the two attributes.

An interesting implication of a favourable permutation is that it reduces the ordinal inequalities in terms of well-being for a very large class of utility functions. Actually, for any increasing utility function, the well-being of the poorer individual increases while that of the richer individual decreases, thereby bringing them closer together on the utility scale. Suppose that the utility function $U(y, h)$ is increasing in both attributes and that $\mathbf{u}$ is obtained from $\mathbf{v}$ by means of a favourable permutation involving two individuals $i$ and $j$ of types $h$ and $k$, respectively, with $h<k$ as defined in (3.17). Then, we have

$$
\begin{aligned}
& U\left(v_{i}^{h}, h\right)<U\left(u_{i}^{h}, h\right)<U\left(u_{j}^{k}, k\right)<U\left(v_{j}^{k}, k\right) ; \text { or } \\
& U\left(v_{i}^{h}, h\right)<U\left(u_{j}^{k}, k\right) \leqq U\left(u_{i}^{h}, h\right)<U\left(v_{j}^{k}, k\right) .
\end{aligned}
$$

This implication of a favourable permutation is reminiscent of the equity condition of Hammond (1976) and in this respect it may be considered an equalising transformation in an ordinal context. We insist on the fact that no use is made of submodularity when deriving the above inequalities which only require that the utility function is increasing in its two arguments.

While it is interesting, this consequence of a favourable permutation is of limited relevance from a practical point of view. As shown by Bosmans and Ooghe (2006) and Miyagishima (2010), the imposition of the equity condition of Hammond (1976) readily precipitates the maximin criterion, which is generally considered too extreme a principle from an ethical point of view. However, favourable permutations have more interesting implications for the inequality of well-being as long as one considers submodular utility functions. Let $\mathbf{u}$ and $\mathbf{v}$ be the heterogeneous distributions associated with the situations $\left(\mathbf{x}^{*} ; \mathbf{a}^{*}\right)$ and $\left(\mathbf{x}^{\circ} ; \mathbf{a}^{\circ}\right)$, respectively, such that $n^{*}(h)=n^{\circ}(h)=n(h)>1$, for all $h \in \mathscr{H}$. Consider the four following statements:

A

$\mathbf{u}$ is obtained from $\mathbf{v}$ by a favourable permutation;

B

$$
\sum_{h=1}^{H} \sum_{i=1}^{n(h)} \psi_{h}\left(u_{i}^{h}\right) \geqq \sum_{h=1}^{H} \sum_{i=1}^{n(h)} \psi_{h}\left(v_{i}^{h}\right), \forall \boldsymbol{\psi}:=\left(\psi_{1}, \ldots, \psi_{H}\right) \in \mathbf{\Psi}
$$

C

$$
\sum_{h=1}^{H} \sum_{i=1}^{n(h)} \phi\left(f\left(u_{i}^{h}\right)+g(h)\right) \geqq \sum_{h=1}^{H} \sum_{i=1}^{n(h)} \phi\left(f\left(v_{i}^{h}\right)+g(h)\right), \forall \phi \text { concave; }
$$

$$
f\left(\mathbf{x}^{*}\right)+g\left(\mathbf{a}^{*}\right) \geq_{L} f\left(\mathbf{x}^{\circ}\right)+g\left(\mathbf{a}^{\circ}\right)
$$

where the functions $f$ and $g$ are increasing. Invoking Lemma 3.3, Hardy-Littlewood-Pólya theorem and the fact that the utility function $\psi(y, h)=\phi(f(y)+g(h))$ is submodular as soon 
as $\phi$ is concave and $f$ and $g$ increasing, we obtain:

$$
A \Longleftrightarrow B \Longrightarrow C \Longleftrightarrow D
$$

There are a number of issues in normative economics that involve comparisons of situations resulting from different rearrangements of distributions. In particular, the chain of implications (4.2) proves to have appealing consequences for comparing the inequalities resulting from different matching and mobility processes.

Matching and inequality We consider two populations of the same size $n$ - men and women - where each population is characterised by a distribution of income. We denote by $\mathbf{u}:=$ $\left(u_{1}, \ldots, u_{n}\right)$ and $\mathbf{v}:=\left(v_{1}, \ldots, v_{n}\right)$ the distributions of income for men and women, respectively, and we assume that no two incomes within distributions $\mathbf{u}$ and $\mathbf{v}$ are the same. The distribution of women's incomes is fixed and their incomes are non-decreasingly arranged which implies that $v_{1}<v_{2}<\cdots<v_{n}{ }^{2}$ We are interested in pairing men and women in such a way that aggregate income inequality is minimised. In order to compare distributions on the basis of inequality, we follow the standard practice and appeal to the standard Lorenz criterion. Given two distributions $\mathbf{x}:=\left(x_{1}, \ldots, x_{n}\right)$ and $\mathbf{y}:=\left(y_{1}, \ldots, y_{n}\right)$ such that $\sum_{i=1}^{n} x_{i}=\sum_{i=1}^{n} y_{i}$, we say that $\mathbf{x}$ Lorenz dominates $\mathbf{y}$, which we write $\mathbf{x} \geq_{L} \mathbf{y}$, if and only if:

$$
\sum_{j=1}^{k} \tilde{x}_{j} \geqq \sum_{j=1}^{k} \tilde{y}_{j}, \forall k=1,2, \ldots, n-1 .
$$

Let $\mathbf{u}^{*}:=\left(u_{1}^{*}, \ldots, u_{n}^{*}\right)$ and $\mathbf{u}^{\circ}:=\left(u_{1}^{\circ}, \ldots, u_{n}^{\circ}\right)$ be two permutations of $\mathbf{u}:=\left(u_{1}, \ldots, u_{n}\right)$. Denote by $\mathbf{u}^{*}+\mathbf{v}:=\left(u_{1}^{*}+v_{1}, \ldots, u_{n}^{*}+v_{n}\right)$ and $\mathbf{u}^{\circ}+\mathbf{v}:=\left(u_{1}^{\circ}+v_{1}, \ldots, u_{n}^{\circ}+v_{n}\right)$ the distributions of aggregate income corresponding to the matchings $\left(\mathbf{u}^{*} ; \mathbf{v}\right)$ and $\left(\mathbf{u}^{\circ} ; \mathbf{v}\right)$, respectively. We deduce immediately from (4.2) that, if $\mathbf{u}^{*}$ is obtained from $\mathbf{u}^{\circ}$ by means of a favourable permutation, then:

$$
\left(\mathbf{u}^{*}+\mathbf{v}\right)>_{L}\left(\mathbf{u}^{\circ}+\mathbf{v}\right)
$$

where $>_{L}$ is the asymmetric component of $\geq_{L}$. Consider a matching where a rich woman is married with a rich man and a poorer woman with a poor man: a switch of men between these two women will result in an unambiguous reduction of inequality in the distribution of the spouses' total income. In particular, the most effective way to reduce income inequality among couples would be to match the richest woman with the poorest man, the second richest woman with the second poorest man, and so on. This provides a theoretical rationale to the conclusion of several studies suggesting that the increasing correlation of spouses' earnings across couple households contributes significantly to widening inequality (see, e.g., Cancian and Reed (1999), Schwartz (2010), Hyslop (2001)).

\footnotetext{
2 These assumptions are made for simplicity and the result would not be modified if we dispensed with these restrictions but the notation would be much heavier.
} 
Exchange mobility We consider a society composed of $n$ dynasties $(n \geqq 2)$, where each dynasty consists of one father and one son. A situation for the society is a $n \times 2$ matrix

$$
\mathbf{x} \equiv\left(\mathbf{x}^{F} ; \mathbf{x}^{S}\right):=\left[\begin{array}{cc}
x_{1}^{F} & x_{1}^{S} \\
\vdots & \vdots \\
x_{h}^{F} & x_{h}^{S} \\
\vdots & \vdots \\
x_{n}^{F} & x_{n}^{S}
\end{array}\right]=\left[\begin{array}{c}
\mathbf{x}_{1} \\
\vdots \\
\mathbf{x}_{h} \\
\vdots \\
\mathbf{x}_{n}
\end{array}\right]
$$

such that $\mathbf{x}_{h}=\left(x_{h}^{F}, x_{h}^{S}\right)$ is the intergenerational distribution of income of dynasty $h$, where $x_{h}^{F} \in \mathscr{S}$ and $x_{h}^{S} \in \mathscr{S}$ are respectively the incomes of dynasty $h$ 's father and son, and where $\mathscr{S}:=\left\{s_{1}, s_{2}, \ldots, s_{m}\right\} \subset \mathscr{D}$ is the set of all possible incomes with $s_{1}<s_{2}<\cdots<s_{m}$. To simplify things, we let $m=n$ and $x_{h}^{F}=s_{h}$, for all $h=1,2, \ldots, n$, which implies that $x_{1}^{F}<x_{2}^{F}<\cdots<x_{n}^{F}$. We also assume that the incomes of children are permutations of parents' incomes which implies that no two children can have the same income. Given the conventions above, two situations $\mathbf{x} \equiv\left(\mathbf{x}^{F} ; \mathbf{x}^{S}\right)$ and $\mathbf{y} \equiv\left(\mathbf{y}^{F} ; \mathbf{y}^{S}\right)$ can only differ to the extent that $\mathbf{x}^{S}$ and $\mathbf{y}^{S}$ are permutations of each other. ${ }^{3}$ Following Atkinson (1981), we say that $\mathbf{x}$ exhibits more mobility than $\mathbf{y}$ if and only if

$$
\sum_{h=1}^{n} V\left(x_{h}^{F}, x_{h}^{S}\right) \geqq \sum_{h=1}^{n} V\left(y_{h}^{F}, y_{h}^{S}\right), \forall V \in \mathscr{V},
$$

where $\mathscr{V}:=\left\{V: \mathscr{D} \times \mathscr{D} \rightarrow \mathbb{R} \mid V_{12}(u, v) \leqq 0, \forall(u, v) \in \mathscr{D} \times \mathscr{D}\right\}$ is the set of admissible dynasty utility functions. It follows from Lemma 3.2 that, if there is greater mobility in $\mathbf{x}$ than in $\mathbf{y}$, then $\mathbf{x}^{S} \geq_{S R O} \mathbf{y}^{S}$, and conversely. Appealing next to Theorem 3.1, this is equivalent to saying that $\mathbf{x}^{S}$ is obtained from $\mathbf{y}^{S}$ by means of a finite sequence of favourable permutations.

The connection between inequality and mobility has been pointed out by Shorrocks (1980, Section 4) who suggested that, the more mobile the society is, the more equally distributed are the dynasties' aggregate incomes. Indeed, consider the class of dynasty utility functions $V(u, v):=\phi(f(u)+g(v))$, where $f(u)+g(v)$ can be interpreted as the net present value of the dynasty's intergenerational income distribution and where $\phi$ measures the value attached to it. Assume in addition that $f$ and $g$ are increasing and that $\phi$ is concave. Then, letting $f\left(\mathbf{z}^{F}\right):=\left(f\left(z_{1}^{F}\right), \ldots, f\left(z_{n}^{F}\right)\right)$ and $g\left(\mathbf{z}^{S}\right):=\left(g\left(z_{1}^{S}\right), \ldots, g\left(z_{n}^{S}\right)\right)$, for $\mathbf{z} \in\{\mathbf{x}, \mathbf{y}\}$, we deduce from (4.2) that, if condition (4.6) holds, then

$$
\left(f\left(\mathbf{x}^{F}\right)+g\left(\mathbf{x}^{S}\right)\right) \geq_{L}\left(f\left(\mathbf{y}^{F}\right)+g\left(\mathbf{y}^{S}\right)\right) .
$$

Therefore, whatever the way we compute the net present values of the intergenerational income distributions - provided that fathers' and sons' incomes contribute positively to it and that their distributions of income are permutations of each other - inequality decreases as mobility increases. ${ }^{4}$

\footnotetext{
${ }^{3}$ Here again we insist on the fact that these assumptions are made for convenience and that they can - to some extent - be relaxed without affecting the results.

${ }^{4}$ A reader, who has some reluctance to accept the assumption that the fathers' and sons' income distributions
} 


\section{Concluding Remarks}

Considering the case where there are two attributes and where the distribution of one of these two attributes is fixed, we have shown that sequential rank order domination of one distribution over another implies that the dominating distribution can be obtained from the dominated one by means of a finite sequence of favourable permutations, and conversely. In a way, the application of the sequential rank order criterion permits one to detect when favourable permutations - and only such operations - are involved in the transformation of distributions. It has been argued that a favourable permutation results in a smoothing of the distribution of the utilities the individuals derive from their endowments in the two attributes. We have provided two examples that involve more or less explicitly favourable permutations and where the application of the sequential rank order criterion proves to be relevant.

As a close inspection of the proof of our main result makes clear, it is not necessary that our first attribute which we have assimilated with income for convenience is of a cardinal nature and our analysis applies equally to distributions of two ordinal attributes. This stems from the fact that the transformations - the favourable permutations - we consider in the paper do not involve transfers of the compensating attribute between individuals. Similarly, the choice of the asymmetrical treatment of the two attributes - income and a categorical variable like health achievement for instance - made in the paper is arbitrary and it can be reversed. This would not affect our main result which builds exclusively on the ordinal properties of our two attributes.

We have implicitly restricted our attention to comparisons of situations where the distributions of the first attribute are permutations of each other and hence have equal means. In other words, we implicitly have assumed that the marginal distributions of the two attributes are fixed as in Tchen (1980) and Epstein and Tanny (1980). While this permits one to stick with a particular way of thinking of inequality - the smoothing impact of favourable permutations mentioned above - we must admit that the applicability of the sequential rank order criterion as defined here to real world comparisons is limited. A natural way of extending our approach would be to relax the assumption according to which the distributions of income have equal means. This raises technical difficulties - one has to find a means of separating favourable permutations from income increments - that are beyond the scope of this paper and it is left for future research. Similarly, one might be tempted to discard the assumption that the distribution of the categorical variable is fixed. But in this case the sequential rank order criterion will have to be abandoned and replaced by the standard first degree stochastic dominance criterion introduced by Atkinson and Bourguignon (1982) in the inequality and welfare literature.

Finally, it must be stressed that the combination of favourable permutations with progressive transfers in an appropriate way allows one to go beyond first degree stochastic -

are permutations of each other, might feel more comfortable if income is replaced by some (ordinal) measure of status as it is typically done by sociologists. Inspection of the argument confirms that this will not change the result. 
equivalently, rank order - dominance. Gravel and Moyes (2012) have shown that the equalising operations that lie behind utilitarian unanimity over the class of concave in income and submodular utility functions can always be decomposed into a favourable permutation followed by a progressive transfer. This confirms that a favourable permutation is a primitive notion that can be regarded as the counterpart of a progressive transfer in a bidimensional setting.

\section{A. Illustration of the Algorithm Used in the Proof of Theorem 3.1}

Consider the two following heterogeneous distributions:

$$
\begin{aligned}
& \mathbf{v}=\left(\mathbf{v}^{1} ; \mathbf{v}^{2} ; \mathbf{v}^{3}\right)=(1,3 ; 2,4,6,7 ; 5,8,9) \\
& \mathbf{u}=\left(\mathbf{v}^{1} ; \mathbf{v}^{2} ; \mathbf{v}^{3}\right)=(6,7 ; 4,5,8,9 ; 1,2,3)
\end{aligned}
$$

We have

$$
\begin{gathered}
\tilde{\mathbf{u}}^{(1)} \equiv(6,7)>(1,3) \equiv \tilde{\mathbf{v}}^{(1)} \\
\tilde{\mathbf{u}}^{(2)} \equiv(4,5,6,7,8,9)>(1,2,3,4,6,7) \equiv \tilde{\mathbf{v}}^{(2)} \\
\tilde{\mathbf{u}}^{(3)} \equiv(1,2,3,4,5,6,7,8,9)=(1,2,3,4,5,6,7,8,9) \equiv \tilde{\mathbf{v}}^{(3)}
\end{gathered}
$$

\begin{tabular}{|c|c|c|c|c|c|c|c|c|c|c|}
\hline & \multicolumn{2}{|c|}{$h=1$} & \multicolumn{4}{|c|}{$h=2$} & \multicolumn{3}{|c|}{$h=3$} & \\
\hline & 1 & 2 & 1 & 2 & 3 & 4 & 1 & 2 & 3 & \\
\hline $\mathbf{u}[1]^{h}:$ & 6 & 7 & 4 & 5 & 8 & 9 & 1 & 2 & 3 & $i^{1}=2 ; s^{1}=4 ; j^{1}=3 ; t^{1}=3$ \\
\hline $\mathbf{w}[1,1]^{h}$ & 1 & 3 & 2 & 4 & 6 & 9 & 5 & 7 & 8 & \\
\hline $\mathbf{v}[1]^{h}:$ & 1 & 3 & 2 & 4 & 6 & 7 & 5 & 8 & 9 & $k_{1}^{1}=2 ; r_{1}^{1}=4$ \\
\hline
\end{tabular}

Thus $\mathbf{u}>_{S R O} \mathbf{v}$.

\section{STEP 1}

We check that:

\begin{tabular}{cccccc}
\hline & $\tilde{\mathbf{u}}[1]^{(h)}$ & & $\tilde{\mathbf{w}}[1,1]^{(h)}$ & & $\tilde{\mathbf{v}}[1]^{(h)}$ \\
\hline$h=1$ & $(6,7)$ & $>$ & $(1,3)$ & $=$ & $(1,3)$ \\
$h=2$ & $(4,5,6,7,8,9)$ & $>$ & $(1,2,3,4,6,9)$ & $>$ & $(1,2,3,4,6,7)$ \\
$h=3$ & $(1,2,3,4,5,6,7,8,9)$ & $=$ & $(1,2,3,4,5,6,7,8,9)$ & $=$ & $(1,2,3,4,5,6,7,8,9)$ \\
\hline
\end{tabular}

We have

$$
\begin{aligned}
& \mathbf{u}[1]_{4}^{2}=\mathbf{z}[1]_{4}^{2} \\
& \mathbf{u}[1]>_{S R O} \mathbf{z}[1]=\mathbf{w}[1,1]>_{S R O} \mathbf{v}[1] \\
& \mathbf{u}[2]:=\mathbf{u}[1] \backslash\left\{u[1]_{4}^{2}\right\} ; \quad \mathbf{v}[2]:=\mathbf{z}[1] \backslash\left\{z[1]_{4}^{2}\right\} ; \\
& \mathbf{u}[2]=(6,7 ; 4,5,8 ; 1,2,3) \neq(1,3 ; 2,4,6 ; 5,7,8)=\mathbf{v}[2]
\end{aligned}
$$

and we proceed to Step 2. 
STEP 2

\begin{tabular}{|c|c|c|c|c|c|c|c|c|c|}
\hline & \multicolumn{2}{|c|}{$h=1$} & \multicolumn{3}{|c|}{$h=2$} & \multicolumn{3}{|c|}{$h=3$} & \\
\hline & 1 & 2 & 1 & 2 & 3 & 1 & 2 & 3 & \\
\hline $\mathbf{u}[2]^{h}:$ & 6 & 7 & 4 & 5 & 8 & 1 & 2 & 3 & $i^{2}=2 ; s^{2}=3 ; j^{2}=3 ; t^{2}=3$ \\
\hline $\mathbf{w}[2,1]^{h}:$ & 1 & 3 & 2 & 4 & 8 & 5 & 6 & 7 & \\
\hline $\mathbf{v}[2]^{h}:$ & 1 & 3 & 2 & 4 & 6 & 5 & 7 & 8 & $k_{1}^{2}=2 ; r_{1}^{2}=3$ \\
\hline
\end{tabular}

We check that:

\begin{tabular}{cccccc}
\hline & $\tilde{\mathbf{u}}[2]^{(h)}$ & & $\tilde{\mathbf{w}}[2,1]^{(h)}$ & & $\tilde{\mathbf{v}}[2]^{(h)}$ \\
\hline$h=1$ & $(6,7)$ & $>$ & $(1,3)$ & $=$ & $(1,3)$ \\
$h=2$ & $(4,5,6,7,8)$ & $>$ & $(1,2,3,4,8)$ & $>$ & $(1,2,3,4,6)$ \\
$h=3$ & $(1,2,3,4,5,6,7,8)$ & $=$ & $(1,2,3,4,5,6,7,8)$ & $=$ & $(1,2,3,4,5,6,7,8)$ \\
\hline
\end{tabular}

We have

$$
\begin{aligned}
& \mathbf{u}[2]_{3}^{2}=\mathbf{z}[2]_{3}^{2} \\
& \mathbf{u}[2]>_{S R O} \mathbf{z}[2]=\mathbf{w}[2,1]>_{S R O} \mathbf{v}[2] \\
& \mathbf{u}[3]:=\mathbf{u}[2] \backslash\left\{u[2]_{3}^{2}\right\} ; \quad \mathbf{v}[3]:=\mathbf{z}[2] \backslash\left\{z[2]_{3}^{2}\right\} \\
& \mathbf{u}[3]=(6,7 ; 4,5 ; 1,2,3) \neq(1,3 ; 2,4 ; 5,6,7)=\mathbf{v}[3]
\end{aligned}
$$

\begin{tabular}{|c|c|c|c|c|c|c|c|c|}
\hline & \multicolumn{2}{|c|}{$h=1$} & \multicolumn{2}{|c|}{$h=2$} & \multicolumn{3}{|c|}{$h=3$} & \\
\hline & 1 & 2 & 1 & 2 & 1 & 2 & 3 & \\
\hline $\mathbf{u}[3]^{h}:$ & 6 & 7 & 4 & 5 & 1 & 2 & 3 & $i^{3}=1 ; s^{3}=2 ; j^{3}=3 ; t^{3}=3$ \\
\hline $\mathbf{w}[3,2]^{h}$ & 1 & 7 & 2 & 3 & 4 & 5 & 6 & \\
\hline $\mathbf{w}[3,1]^{h}$ & 1 & 3 & 2 & 7 & 4 & 5 & 6 & $k_{2}^{3}=1 ; r_{2}^{3}=2$ \\
\hline $\mathbf{v}[3]^{h}:$ & 1 & 3 & 2 & 4 & 5 & 6 & 7 & $k_{1}^{3}=2 ; r_{1}^{3}=2$ \\
\hline
\end{tabular}

and we proceed to Step 3.

\section{STEP 3}

We check that:

\begin{tabular}{cccccccc}
\hline & $\tilde{\mathbf{u}}[3]^{(h)}$ & & $\tilde{\mathbf{w}}[3,2]^{(h)}$ & & $\tilde{\mathbf{w}}[3,1]^{(h)}$ & & $\tilde{\mathbf{v}}[3]^{(h)}$ \\
\hline$h=1$ & $(6,7)$ & $>$ & $(1,7)$ & $>$ & $(1,3)$ & $=$ & $(1,3)$ \\
$h=2$ & $(4,5,6,7)$ & $>$ & $(1,2,3,7)$ & $=$ & $(1,2,3,7)$ & $>$ & $(1,2,3,4)$ \\
$h=3$ & $(1,2,3,4,5,6,7)$ & $=$ & $(1,2,3,4,5,6,7)$ & $=$ & $(1,2,3,4,5,6,7)$ & $=$ & $(1,2,3,4,5,6,7)$ \\
\hline
\end{tabular}

We have

$$
\begin{aligned}
& \mathbf{u}[3]_{2}^{1}=\mathbf{z}[3]_{2}^{1} \\
& \mathbf{u}[3]>_{S R O} \mathbf{z}[3]=\mathbf{w}[3,2]>_{S R O} \mathbf{w}[3,1]>_{S R O} \mathbf{v}[3] \\
& \mathbf{u}[4]:=\mathbf{u}[3] \backslash\left\{u[3]_{2}^{1}\right\} ; \quad \mathbf{v}[4]:=\mathbf{z}[3] \backslash\left\{z[3]_{2}^{1}\right\} \\
& \mathbf{u}[4]=(6 ; 4,5 ; 1,2,3) \neq(1 ; 2,3 ; 4,5,6)=\mathbf{v}[4]
\end{aligned}
$$

and we proceed to Step 4. 


\section{STEP 4}

\begin{tabular}{|c|c|c|c|c|c|c|c|}
\hline & \multirow{2}{*}{$\begin{array}{c}h=1 \\
1\end{array}$} & \multicolumn{2}{|c|}{$h=2$} & \multicolumn{3}{|c|}{$h=3$} & \\
\hline & & 1 & 2 & 1 & 2 & 3 & \\
\hline $\mathbf{u}[4]^{h}:$ & 6 & 4 & 5 & 1 & 2 & 3 & $i^{4}=1 ; s^{4}=1 ; j^{4}=3 ; t^{4}=3$ \\
\hline $\mathbf{w}[4,2]^{h}$ & 6 & 1 & 2 & 3 & 4 & 5 & \\
\hline $\mathbf{w}[4,1]^{h}$ & 1 & 2 & 6 & 3 & 4 & 5 & $k_{2}^{4}=1 ; r_{2}^{4}=1$ \\
\hline $\mathbf{v}[4]^{h}:$ & 1 & 2 & 3 & 4 & 5 & 6 & $k_{1}^{4}=2 ; r_{1}^{4}=2$ \\
\hline
\end{tabular}

We check that:

\begin{tabular}{cccccccc}
\hline & $\tilde{\mathbf{u}}[4]^{(h)}$ & & $\tilde{\mathbf{w}}[4,2]^{(h)}$ & & $\tilde{\mathbf{w}}[4,1]^{(h)}$ & & $\tilde{\mathbf{v}}[4]^{(h)}$ \\
\hline$h=1$ & $(6)$ & $=$ & $(6)$ & $>$ & $(1)$ & $=$ & $(1)$ \\
$h=2$ & $(4,5,6)$ & $>$ & $(1,2,6)$ & $=$ & $(1,2,6)$ & $>$ & $(1,2,6)$ \\
$h=3$ & $(1,2,3,4,5,6)$ & $=$ & $(1,2,3,4,5,6)$ & $=$ & $(1,2,3,4,5,6)$ & $=$ & $(1,2,3,4,5,6)$ \\
\hline
\end{tabular}

We have

$$
\begin{aligned}
& \mathbf{u}[4]_{1}^{1}=\mathbf{z}[4]_{1}^{1} \\
& \mathbf{u}[4]>_{S R O} \mathbf{z}[4]=\mathbf{w}[4,2]>_{S R O} \mathbf{w}[3,1]>_{S R O} \mathbf{v}[4] \\
& \mathbf{u}[5]:=\mathbf{u}[4] \backslash\left\{u[4]_{1}^{1}\right\} ; \quad \mathbf{v}[5]:=\mathbf{z}[4] \backslash\left\{z[4]_{1}^{1}\right\} \\
& \mathbf{u}[5]=(4,5 ; 1,2,3) \neq(1,2 ; 3,4,5)=\mathbf{v}[5]
\end{aligned}
$$

\begin{tabular}{|c|c|c|c|c|c|c|}
\hline & \multicolumn{2}{|c|}{$h=2$} & \multicolumn{3}{|c|}{$h=3$} & \\
\hline & 1 & 2 & 1 & 2 & 3 & \\
\hline $\mathbf{u}[5]^{h}:$ & 4 & 5 & 1 & 2 & 3 & $i^{5}=2 ; s^{5}=2 ; j^{5}=3 ; t^{5}=3$ \\
\hline $\mathbf{w}[5,1]^{h}$ & 1 & 5 & 2 & 3 & 4 & \\
\hline $\mathbf{v}[5]^{h}:$ & 1 & 2 & 3 & 4 & 5 & $k_{1}^{5}=2 ; r_{1}^{5}=2$ \\
\hline
\end{tabular}

and we proceed to Step 5.

\section{STEP 5}

We check that:

\begin{tabular}{cccccc}
\hline & $\tilde{\mathbf{u}}[5]^{(h)}$ & & $\tilde{\mathbf{w}}[5,1]^{(h)}$ & & $\tilde{\mathbf{v}}[5]^{(h)}$ \\
\hline$h=2$ & $(4,5)$ & $>$ & $(1,5)$ & $>$ & $(1,2)$ \\
$h=3$ & $(1,2,3,4,5)$ & $=$ & $(1,2,3,4,5)$ & $=$ & $(1,2,3,4,5)$ \\
\hline
\end{tabular}

We have

$$
\begin{aligned}
& \mathbf{u}[5]_{2}^{2}=\mathbf{z}[5]_{2}^{2} \\
& \mathbf{u}[5]>_{S R O} \mathbf{z}[5]=\mathbf{w}[5,1]>_{S R O} \mathbf{v}[5] \\
& \mathbf{u}[6]:=\mathbf{u}[5] \backslash\left\{u[5]_{2}^{2}\right\} ; \quad \mathbf{v}[6]:=\mathbf{z}[5] \backslash\left\{z[5]_{2}^{2}\right\} \\
& \mathbf{u}[6]=(4 ; 1,2,3) \neq(1 ; 2,3,4)=\mathbf{v}[6]
\end{aligned}
$$

and we proceed to Step 6. 


\section{STEP 6}

\begin{tabular}{|c|c|c|c|c|c|}
\hline & \multirow{2}{*}{$\begin{array}{c}h=2 \\
1\end{array}$} & \multicolumn{3}{|c|}{$h=3$} & \\
\hline & & 1 & 2 & 3 & \\
\hline $\mathbf{u}[6]^{h}:$ & 4 & 1 & 2 & 3 & $i^{6}=2 ; s^{6}=1 ; j^{6}=3 ; t^{3}=3$ \\
\hline $\mathbf{w}[6,1]^{h}$ & 4 & 1 & 2 & 3 & \\
\hline $\mathbf{v}[6]^{h}:$ & 1 & 2 & 3 & 4 & $k_{1}^{6}=2 ; r_{1}^{6}=1$ \\
\hline
\end{tabular}

We check that:

\begin{tabular}{lclclc}
\hline & $\tilde{\mathbf{u}}[6]^{(h)}$ & & $\tilde{\mathbf{w}}[6,1]^{(h)}$ & & $\tilde{\mathbf{v}}[6]^{(h)}$ \\
\hline$h=2$ & $(4)$ & $=$ & $(4)$ & $>$ & $(1)$ \\
$h=3$ & $(1,2,3,4)$ & $=$ & $(1,2,3,4)$ & $=$ & $(1,2,3,4)$ \\
\hline
\end{tabular}

We have

$$
\begin{aligned}
& \mathbf{u}[6]_{1}^{2}=\mathbf{z}[6]_{1}^{2} \\
& \mathbf{u}[6]=\mathbf{z}[6]=\mathbf{w}[6,1]>_{S R O} \mathbf{v}[6] \\
& \mathbf{u}[7]:=\mathbf{u}[6] \backslash\left\{u[6]_{1}^{2}\right\} ; \quad \mathbf{v}[7]:=\mathbf{z}[6] \backslash\left\{z[6]_{1}^{2}\right\} \\
& \mathbf{u}[7]=(4 ; 1,2,3)=(4 ; 1,2,3)=\mathbf{v}[7]
\end{aligned}
$$

and we are home.

\section{References}

Atkinson, A. B. (1981). The measurement of economic mobility. In P. Eijgelshoven and L. van Gemerden, editors, Inkomensverdeling en Openbare Financiën: Opstellen voor Jan Pen, pages 9-24. Uitgeverij Het Spectrum, Utrecht/Antwerpen.

Atkinson, A. B. and Bourguignon, F. (1982). The comparison of multidimensioned distributions of economic status. Review of Economic Studies, 49, 183-201.

Atkinson, A. B. and Bourguignon, F. (1987). Income distributions and differences in needs. In G. Feiwel, editor, Arrow and the Foundations of the Theory of Economic Policy, pages 350-370. MacMillan, New York.

Berge, C. (1963). Topological Spaces, Including a Treatment of Multi-Valued Functions, Vector Spaces and Convexity. Oliver and Boyd, Edinburgh.

Bosmans, C. and Ooghe, E. (2006). A characterization of maximin. Katholieke Universiteit Leuven.

Cancian, M. and Reed, D. (1999). The impact of wives' earnings on income inequality: Issues and estimates. Demography, 36, 173-184.

Dasgupta, P., Sen, A. K., and Starrett, D. (1973). Notes on the measurement of inequality. Journal of Economic Theory, 6, 180-187. 
Epstein, L. G. and Tanny, S. (1980). Increasing generalized correlation: A definition and some economic consequences. Canadian Journal of Economics, 13, 16-34.

Fishburn, P. C. and Vickson, R. G. (1978). Theoretical foundations of stochastic dominance. In G. Whitmore and M. Findlay, editors, Stochastic Dominance, pages 39-113. Lexington Books, Lexington, MA.

Fleurbaey, M. (2008). Fairness, Responsibility and Welfare. Oxford University Press, Oxford.

Gravel, N. and Moyes, P. (2012). Ethically robust comparisons of bidimensional distributions with an ordinal attribute. Journal of Economic Theory, 147, 1384-1426.

Hammond, P. J. (1976). Equity in two person situations: Some consequences. Econometrica, 47, 1127-1135.

Hardy, G. H., Littlewood, J. E., and Pólya, G. (1934). Inequalities. Cambridge University Press, Cambridge.

Hyslop, D. R. (2001). Rising U.S. earnings inequality and family labor supply: The covariance structure of intrafamily earnings. American Economic Review, 91, 755-777.

Marshall, A. W. and Olkin, I. (1979). Inequalities: Theory of Majorization and its Applications. Academic Press, New York.

Miyagishima, K. (2010). A characterization of the maximin social ordering. Economics Bulletin, 30(2), 1278-1282.

Moyes, P. (2012). Comparisons of heterogeneous distributions and dominance criteria. Journal of Economic Theory, 147, 1351-1383.

Muller, C. and Trannoy, A. (2012). Multidimensional inequality comparisons: A compensation perspective. Journal of Economic Theory, 147, 1427-1449.

Roemer, J. E. (1996). Theories of Distributive Justice. Harvard University Press, Cambridge, USA.

Saposnik, R. (1981). Rank-dominance in income distributions. Public Choice, 36, 147-151.

Schwartz, C. R. (2010). Earnings inequality and the changing association between spouses' earnings. American Journal of Sociology, 115, 1524-1557.

Shorrocks, A. F. (1980). Income stability in the United States. In N. A. Klevmarken and J. A. Lybeck, editors, The Statics and Dynamics of Income, pages 175-194. Tieto Limited, Clevedon.

Tchen, A. H. (1980). Inequalities for distributions with given marginals. The Annals of Probability, 8, 814-827. 


\title{
Cahiers du GREThA Working papers of GREThA
}

\author{
GREThA UMR CNRS 5113 \\ Université Montesquieu Bordeaux IV \\ Avenue Léon Duguit \\ 33608 PESSAC - FRANCE \\ Tel : +33 (0)5.56.84.25.75 \\ Fax : +33 (0)5.56.84.86.47 \\ http://gretha.u-bordeaux4.fr/
}

\begin{abstract}
Cahiers du GREThA (derniers numéros - last issues)
2012-27 : OUEDRAOGO Boukary, Population et environnement : Cas de la pression anthropique sur la forêt périurbaine de Gonsé au Burkina Faso.

2012-28 : OUEGRAOGO Boukary, FERRARI Sylvie, Incidence of forest income in reducing poverty and inequalities: Evidence from forest dependant households in managed forest' areas in Burkina Faso

2012-29 : PEZZONI Michele, LISSONI Francesco, TARASCONI Gianluca, How To Kill Inventors: Testing The Massacrator ${ }^{\circ}$ Algorithm For Inventor Disambiguation

2013-01 : KAMMOUN Olfa, RAHMOUNI Mohieddine, Intellectual Property Rights, Appropriation Instruments and Innovation Activities: Evidence from Tunisian Firms

2013-02 : BLANCHETON Bertrand, NENOVSKY Nikolay, Protectionism and Protectionists Theories at the Balkans in the Interwar Period

2013-03: AUGERAUD-VERON Emmanuelle, LEANDRI Marc, Optimal pollution control with distributed delays

2013-04 : MOUYSSET Lauriane, DOYEN Luc, PEREAU Jean-Christophe, JIGUET Frédéric, A double benefit of biodiversity in agriculture

2013-05 : OUEDRAOGO Boukary, HASSANE Mamoudou Co-intégration et causalité entre PIB, emploi et consommation d'énergie: évidence empirique sur les pays de l'UEMOA

2013-06 : MABROUK Fatma, À la recherche d'une typologie des migrants de retour :le cas des pays du Maghreb

2013-07 : LASKOWSKI Natacha, Optimal allocation of wetlands: Study on conflict between agriculture and fishery

2013-08 : OUEDRAOGO Boukary, Acceptation et usages éducatifs des Technologies de I'Information et de la Communication (TIC) par les professeurs de l'Université de Ouagadougou (Burkina Faso) : une application du modèle TUAUT
\end{abstract}

2013-09: FRIGANT Vincent, Une comparaison de l'internationalisation des chaînes d'approvisionnement automobiles allemande et française

2013-10 : MOYES Patrick, Rearrangements and Sequential Rank Order Dominance

La coordination scientifique des Cahiers du GREThA est assurée par Sylvie FERRARI et Vincent FRIGANT. La mise en page est assurée par Anne-Laure MERLETTE. 